Article

\title{
Chemical Composition of Myrtle (Myrtus communis L.) Berries Essential Oils as Observed in a Collection of Genotypes
}

\author{
Marianna Usai ${ }^{1,+}$, Mauro Marchetti ${ }^{2,+}$, Nicola Culeddu ${ }^{2,+}$ and Maurizio Mulas ${ }^{3, *,+(D)}$ \\ 1 Department of Chemistry and Pharmacy, University of Sassari, Via Muroni 23/ A, I-07100 Sassari, Italy; \\ dsfusai@uniss.it \\ 2 Institute of Biomolecular Chemistry, National Research Council (CNR), Trav. La Crucca 3, \\ 07100 Sassari, Italy; mauro@ss.cnr.it (M.M.); nicola.culeddu@icb.cnr.it (N.C.) \\ 3 Department of Agriculture, University of Sassari, Via De Nicola 9, I-07100 Sassari, Italy \\ * Correspondence: mmulas@uniss.it; Tel.: +39-079-229-334; Fax: +39-079-229-337 \\ + The authors contributed equally to this work.
}

Academic Editor: Luca Forti

Received: 27 August 2018; Accepted: 27 September 2018; Published: 29 September 2018

check for updates

\begin{abstract}
Myrtle (Myrtus communis L.) is a shrub spontaneously growing in the Mediterranean area. The leaf and fruit content of essential oils and phenolic compounds justify the wide use of the plant as medicinal and aromatic. Because of overexploitation of wild plants, a domestication process is in progress in different regions and the influence of the genotype variability on the chemical composition of fruit essential oils may be useful to breeding programs. Consequently, the analysis performed on a selected group of candidate clones growing in the same field collection in Sardinia is the object of this report. Forty-seven selections provided fully ripe fruits for essential oil extraction by hydrodistillation and Gas Chromatography-Mass Spectrometry (GC-MS) analysis. Only five candidate clones showed white fruits. The highest yield of essential oil was observed in the LAC31 genotype with $0.55 \mathrm{~g} \cdot \mathrm{kg}^{-1}$, while the samples BOS1, MON5, RUM4, RUM10, V4 and V8 showed values above $0.20 \mathrm{~g} \cdot \mathrm{kg}^{-1}$ and most of the genotypes under $0.10 \mathrm{~g} \cdot \mathrm{kg}^{-1}$. Geranyl acetate was the compound with the highest relative abundance. The second compound for relative abundance was the 1,8-cineole. Other compounds with high relative abundance were $\alpha$-terpinyl acetate, methyleugenol, linalool, $\alpha$-terpineol, $\beta$-caryophyllene, $\alpha$-humulene, Trans-caryophyllene oxide, and humulene epoxide II.
\end{abstract}

Keywords: Myrtaceae family; myrtle fruit; volatile composition; GC-MS; genetic variability

\section{Introduction}

Myrtle (Myrtus communis L.) grows wild in the Mediterranean basin up to $800 \mathrm{~m}$ above sea level. Myrtle prospers in mild climates, fears frost but not drought, and prefers sandy, loose permeable soil with neutral or sub acid reaction. It is common in the Mediterranean maquis. In Sardinia and Corsica, it is a part of low Mediterranean maquis [1-5].

There are many scientific articles on the composition and biological activities of Myrtus communis; most studies on myrtle have focused on its volatile fraction. Due to its importance in the perfume and flavor industry, the chemical composition of myrtle essential oils was previously studied mainly in leaves from different geographic areas: Italy, Sardinia, Corsica, Tunisia, Algeria, Greece, Cyprus, Montenegro, Croatia, and Iran [6-29].

The presence of essential oils in all tissues is of fundamental importance to determine the antioxidant, antibiotic and antimutagenic properties of the myrtle biomass $[21,22,24,30]$. Several 
studies have indicated the activities of Myrtus chemical components [31-33]. Less information is available on myrtle essential oil from flowers $[13,17,23,34]$.

In addition, less investigation was performed on the essential oils of berries and few papers have been published on these topics [23,34-37].

Boelens et al. [34] analyzed the hydrodistilled oils coming from Spanish wild-growing unripe and ripe fruits. Eighty components have been identified and quantified. The yields of the hydrodistilled oils were obtained for unripe and ripe fruits: $0.5 \%$ and $0.02 \%$ respectively. They found that during ripening the concentration of the main constituents changed: e.g., 1,8-cineole increased from $19.5 \%$ to $61.5 \%$ while myrtenyl acetate decreased from $33.0 \%$ to $0.1 \%$.

Jerkovic et al. [23] studied the Croatian myrtle fruit oils along the year and found that myrtenyl acetate (12.2-33.2\%), 1,8-cineole + limonene (10.9-21.1\%), $\alpha$-pinene (4.0-15.3\%), and linalool (4.7-7.7\%) were the major constituents. Among them, linalool showed minimal quantitative changes. During the collecting period, high concentration of myrtenyl acetate was detected in September, while the concentrations of the four other quantitatively important compounds were highest in February, when the concentration of myrtenyl acetate was lowest. In this period, the fruit oil yields varied from 0.03 to $0.13 \%$.

Pereira et al. [36] studied the composition of essential oil from Portuguese myrtle through the vegetative cycle. They found that Portuguese essential oils of myrtle berries are characterized by high content of limonene $+1,8$-cineole $(25.9 \%)$ and myrtenyl acetate $(6.6 \%)$. $\alpha$-pinene $(9.7 \%)$ and linalool $(36.5 \%)$ are also present at high level. These results indicate that Portuguese myrtle belongs to the group of myrtle genotypes, which characterized by the presence of myrtenyl acetate as one of the major components.

Messaoud et al. [37] report of some Gas Chromatography-Mass Spectrometry (GC-MS) analyses of essential oils extracts from mature dark blue and white berries of Tunisian Myrtus communis samples growing at the same site, which allowed the identification of 33 chemical components. The oils from dark blue fruits showed high percentages of $\alpha$-pinene (11.1\%), linalool (11.6\%), $\alpha$-terpineol $(15.7 \%)$, methyl eugenol (6.2\%), and geraniol (3.7\%). Myrtenyl acetate $(20.3 \%)$ was found to be the major compound in the oils from white berries.

Brada et al. [38] studied the Algerian myrtle essential oil and the yield obtained from berries was $0.1 \%$. Twenty-four constituents were identified, representing $89.5 \%$ of the berry oil analysis, the main components being: linalool (36.2\%), followed by estragole (18.4\%) and 1,8-cineole $(11.4 \%)$. The oxygenated monoterpenes were the predominant chemical group $(71.2 \%)$, followed by the sesquiterpenoids (16\%). Monoterpenes $(1.7 \%)$ and oxygenated sesquiterpenes $(0.4 \%)$ were very low. Berry oil is characterized by a great amount of linalool, estragole, 1,8 cineole and an appreciable amount of bergamotene and E-caryophyllene.

Kafkas et al. [39] studied the volatile compounds of white and black myrtle from Turkey. Seven samples (identified by numbers) were collected in two different stations and fruit volatiles were extracted by HS/SPME. Thirty-one volatile compounds were identified in fruits. The lowest hexanal percentage was detected in type 2, while the highest was detected in type 4 . Four ester compounds were detected in white myrtle types, whereas no ester was identified in black myrtle types. Linanyl-butyrate and linanyl-acetate were detected with higher percentages. Alcohols were detected as major compounds except type 16, whereas, terpenes compounds were detected as major compounds in type 16 .

Among the detected terpene compounds, $\alpha$-pinene was the major compound. Limonene was detected in white myrtle types, whereas, this compound was not detected in black types. Eucalyptol was detected in higher percentages in black myrtle (types 4 and 5, respectively) compared to the white types $(2,3,8,16$ and 1 , respectively).

Mazza in 1983 [35] make the first GC-MS investigation on the volatile components of myrtle berries from Sardinian myrtle berries. He gave a detailed picture of the volatile components of myrtle berries analyzing the methanol extract of berries from Sardinia after centrifugation with water and 
extraction with pentane-methylene chloride that was used for GC-MS analyses. An alcoholic extract (60\% ethanol) and commercial samples of liqueurs were also analyzed.

The extract obtained with solvent showed that $\alpha$-pinene, limonene and 1,8-cineole represent $72 \%$ of the volatile fraction. Eleven hydrocarbons have been identified. Alcohols are $11 \%$ of volatile fraction and linalool and $\alpha$-terpineol (being the most abundant) together reach $57 \%$ of total.

Tuberoso et al. [11] investigated the chemical composition of volatiles in Sardinian myrtle alcoholic extracts and essential oils. Although the content of monoterpenes represented $65.7-89.1 \%$ of the oil samples, some chemical constituents were remarkably different. For example, $\alpha$-pinene ranged from $18.2 \%$ to $38.9 \%, \delta$-3-carene from 0.0 to $6.1 \%$, p-cymene from $0.1 \%$ to $10.3 \%$, limonene widely ranged from $3.7 \%$ to $44.5 \%, 1,8$-cineole from $5.8 \%$ to $24.8 \%, \gamma$-terpinene from $0.5 \%$ to $5.8 \%$; terpinolene from $0.0 \%$ to $5.9 \%$. Linalool widely ranged from $0.4 \%$ to $14.7 \%$, terpenyl acetate from $0.1 \%$ to $5.4 \%$, and geranyl acetate from $0.2 \%$ to $13.0 \%$. The berries showed a moderate amount of sesquiterpenes representing $5.0 \%$ of the entire oil at the most.

Since ancient times, myrtle has been used as a medicinal plant. In Sardinia, it is very common the production of a myrtle liqueur [5]. Considering the high economic importance of myrtle industry in Sardinia the characterization of the genetic variability in wild and domesticated accessions may be a fundamental contribute to the breeding of the species [40-42].

Melito et al. in 2013 and in 2017 [40-42] studied genotypic variation and genetic diversity that were characterized using standard population genetics approaches. The level of genetic variability was high. The genetic data were compatible with the notion that myrtle has a mixed pollination system, including both out-pollination by insects and self-pollination. The candidate cultivars may represent an appropriate basis for directed breeding. All these selections are cultivated in Fenosu (Oristano) experimental field and represent a wide population usable to investigate chemical variations in these genotypes. In this field the production of different chemical profiles, in all parts of these plants, are regulated only by genetic differences because environmental condition is the same for all populations. In this view, considering the low level of information on the chemical composition of myrtle berries essential oils and the importance of these to determine the flavor of myrtle industry products, we investigated the chemical variation of berries essential oils with the aim to standardize the potential use of every clone selection.

\section{Results and Discussion}

In our studies, we consider 47 different candidate clones: only five of leucocarpa varietas and the most part of melanocarpa varietas. The yield of essential oil of the berries was highly variable (Figure 1). Six candidate clones did not have enough quantity of fruits to guarantee a correct random harvesting and oil yield. LAC10 and TEL2, do not seem to produce essential oils in appreciable amount, while CPT5, RUM15, RUM13 and 6/2 gave yields around $0.005 \mathrm{~g} \cdot \mathrm{kg}^{-1}$, but the investigation has not resulted in a GC chromatogram associable to typical components of essential oils but only to hydrocarbons probably attributable to waxes. The highest yield of essential oil was observed in the LAC31 genotype with $0.55 \mathrm{~g} \cdot \mathrm{kg}^{-1}$, while the samples BOS1, MON5, RUM4, RUM10, V4 and V8 showed values above $0.20 \mathrm{~g} \cdot \mathrm{kg}^{-1}$ and most of the genotypes under $0.10 \mathrm{~g} \cdot \mathrm{kg}^{-1}$.

GC-MS analysis of essential oils resulted in the detection of 92 compounds that are showed in the Tables 1-4. The main components were: geranyl acetate for 13 genotypes; 1,8-cineole for 7 genotypes; $\alpha$-terpinyl-acetate for 4 genotypes; linalool, $\alpha$-humulene, Trans-caryophyllene oxide and $\beta$-caryophyllene for 3 genotypes; limonene for 2 genotypes; $\alpha$-terpineol, bornyl acetate and humulene epoxide II respectively for 1 genotype each. $\beta$-caryophyllene was the compound present in all the genotypes and methyl eugenol in 40 selections.

The compounds present only in one genotype with small quantities were the 2-methylbutanoic acid, 2 methylpropil ester, the $p$-mentha-1(7),8-diene, the linalyl acetate, the $\beta$-bisabolene, the ledol and the leptospermone iso. 
Geranyl acetate was the compound with the highest relative abundance in all the population of candidate clones. It was present in 35 genotypes and the compound with the highest percentage in one essential oil, with the $50.95 \%$ in the candidate clone V5. The presence of geranyl acetate in the essential oils of the studied population gives a strong characterization to the same.

The second compound for relative abundance was the 1,8-cineole. It was present in 25 genotypes and the highest percentage was $43.26 \%$ in the candidate clone V4. Other compounds with high relative abundance were $\alpha$-terpinyl acetate with the maximum of $23.56 \%$ in RUB95, methyleugenol with $19.66 \%$ in the sample LAC11, linalool with $35.10 \%$ in ISL3, $\alpha$-terpineol with $23.21 \%$ in the RUM14 genotype, $\beta$-caryophyllene with $35.00 \%$ in MON5, $\alpha$-humulene with $24.72 \%$ in LAC31, Trans-caryophyllene oxide with $25.69 \%$ in ORO2, and humulene epoxide II with $15.43 \%$ in ISL1.

The five genotypes with white berries showed limonene, 1,8-cineole, $\alpha$-terpinyl acetate, and $\alpha$-terpineol as main components but this chemotype was not exclusive of the candidate clones with white fruits.

The studied population of candidate clones had a high variability among genotypes and data on chemical composition of essential oils were quite different with respect to previously published data on samples from Sardinia and different other areas of Mediterranean region [25,34-39]. The recurrent association of main compounds was among $\alpha$-terpinyl acetate, geranyl acetate, methyl eugenol and $\alpha$-terpineol, as markedly evidenced in the candidate clones RUM6, BUB95, RUM20, CPT4, V9, V12, LAC1, ORS2, ORS3, SIN2, and PSF1 and only partially reported by other authors for Sardinia myrtle berry essential oils [11,35]. Other associations of main compounds were that of $\alpha$-pinene, $p$-cymene, limonene, and 1,8-cineole, as reported for the selections RUB3, V8, RUM10, CPT6, V4, and BOS1; and that of limonene, 1,8-cineole, linalool, and geranyl acetate, characterizing RUM13, RUM14, and RUM4B. The association between linalool and geranyl acetate was also observed as characteristic of V17, V19, and V20.

Many of the most important components of these possible chemotypes have been previously reported as part of myrtle berries essential oils [11,23,34-39]. However, some aspects appeared as new and not previously reported. Firstly, the relative scarcity of $\alpha$-pinene and linalool, always indicated by previous studies as two of the most abundant compounds. Furthermore, the absence or low content of myrtenyl acetate is a result that differentiates our study from all the previous findings [11,23,34-39].

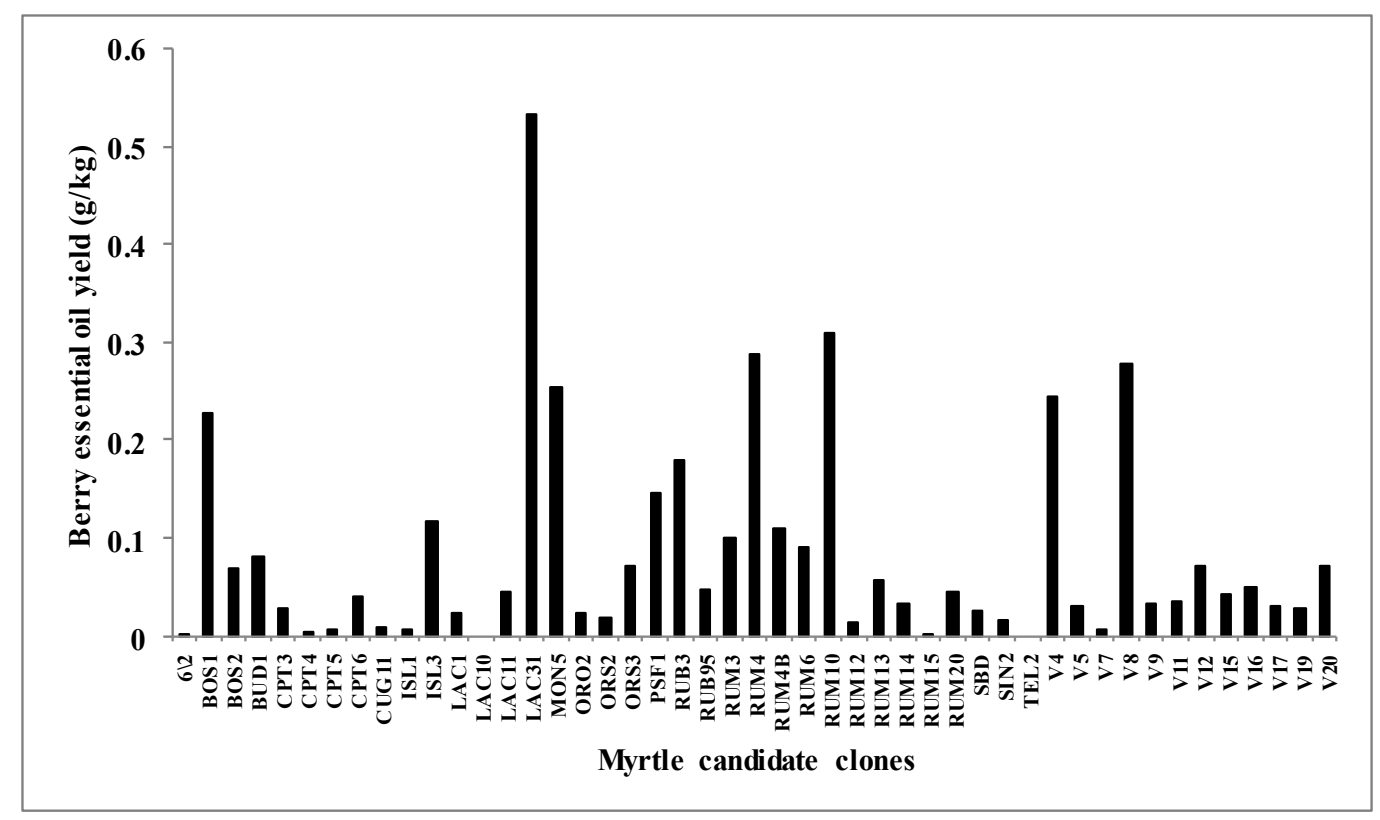

Figure 1. Yield of essential oils from 47 candidate clones of berries of M. communis L. The least significant difference value was 0.064 according to the application of Tukey's test at $p \leq 0.05$ level. 
Table 1. Chemical composition of berry essential oil of ten (n. 1-10) myrtle genotypes.

\begin{tabular}{|c|c|c|c|c|c|c|c|c|c|c|c|c|c|c|c|}
\hline & & & Genotype Number & 1 & 2 & 3 & 4 & 5 & 6 & 7 & 8 & 9 & 10 & $\mathrm{ID}^{\mathrm{z}}$ & Ref. \\
\hline & & & Selection & RUM6 & RUM14 & RUB3 & RUB95 & v8 & RUM3 & RUM4 & RUM4B & RUM10 & RUM12 & & \\
\hline $\mathbf{R t}$ & $\begin{array}{c}\text { KI } \\
\text { Apolar } \\
\text { Lit. }\end{array}$ & $\begin{array}{c}\text { KI } \\
\text { apolar }\end{array}$ & Compound & $\%$ & $\%$ & $\%$ & $\%$ & $\%$ & $\%$ & $\%$ & $\%$ & $\%$ & $\%$ & & \\
\hline 17.88 & 939 & 937 & $\alpha$-pinene & $0.52 \pm 0.02$ & & $11.88 \pm 0.77$ & & $10.32 \pm 0.26$ & & $2.90 \pm 0.16$ & $1.22 \pm 0.02$ & $8.54 \pm 0.47$ & & Std & \\
\hline 21.40 & 986 & 985 & butanoic acid, 2-methyl-2-2-methylpropyl ester & & & & & & & & & $0.28 \pm 0.03$ & & MS-RI & [43] \\
\hline 21.6 & 1002 & 1001 & $\alpha$-phellandrene & & & & & & & & & & & Std & \\
\hline 21.93 & 1002 & 1002 & $\delta$-2-carene & & & $1.56 \pm 0.08$ & & $2.46 \pm 0.41$ & & & $0.57 \pm 0.04$ & $2.15 \pm 0.09$ & $0.05 \pm 0.01$ & Std & \\
\hline 22.25 & 1005 & 1004 & pseudolimonene & & & & & & & & & & & MS-RI & [44] \\
\hline 22.66 & 1025 & 1021 & -cymene & $2.80 \pm 0.11$ & & $4.44 \pm 0.05$ & $1.23 \pm 0.06$ & $9.70 \pm 0.13$ & $1.75 \pm 0.12$ & $4.86 \pm 0.28$ & $2.77 \pm 0.19$ & $8.66 \pm 0.43$ & $0.02 \pm 0.01$ & Std & \\
\hline 22.90 & 1031 & 1029 & limonene & $3.04 \pm 0.16$ & $1.54 \pm 0.08$ & $7.34 \pm 0.32$ & $1.39 \pm 0.08$ & $26.80 \pm 1.24$ & $1.19 \pm 0.09$ & $4.12 \pm 0.22$ & $19.18 \pm 0.97$ & $20.91 \pm 1.04$ & & Std & \\
\hline 23.07 & 1035 & 1031 & 1,8-cineole & $17.20 \pm 0.47$ & $5.34 \pm 0.39$ & $36.41 \pm 0.76$ & $9.38 \pm 0.43$ & $26.67 \pm 1.37$ & $11.50 \pm 0.78$ & $25.95 \pm 0.89$ & $8.49 \pm 0.17$ & $22.69 \pm 1.29$ & & Std & \\
\hline 24.49 & 1060 & 1057 & $\gamma$-terpinene & $0.64 \pm 0.03$ & & $1.92 \pm 0.10$ & $0.39 \pm 0.03$ & $0.95 \pm 0.05$ & $0.80 \pm 0.03$ & $1.82 \pm 0.03$ & $1.21 \pm 0.06$ & $0.86 \pm 0.05$ & & Std & \\
\hline 26.08 & 1089 & 1063 & $\alpha$-terpinolene & & & $0.94 \pm 0.06$ & & & & & $0.69 \pm 0.02$ & & & Std & \\
\hline 26.55 & 1097 & 1094 & linalool & $2.32 \pm 0.09$ & $6.30 \pm 0.46$ & $2.03 \pm 0.11$ & $2.89 \pm 0.15$ & $6.39 \pm 0.39$ & $14.93 \pm 0.86$ & $13.23 \pm 0.45$ & $11.48 \pm 1.04$ & $7.60 \pm 0.14$ & & Std & \\
\hline 26.75 & 1112 & 1108 & $\mathrm{n}$-amyl isovalerate & $3.24 \pm 0.18$ & $1.83 \pm 0.03$ & $3.26 \pm 0.17$ & $3.41 \pm 0.16$ & $3.64 \pm 0.26$ & $3.83 \pm 0.12$ & $4.24 \pm 0.09$ & $1.38 \pm 0.05$ & $3.79 \pm 0.17$ & & MS-RI & [45] \\
\hline 28.81 & 1113 & 1115 & Trans-pinocarveol & $0.89 \pm 0.05$ & & $0.50 \pm 0.02$ & $0.83 \pm 0.09$ & & & & & $0.35 \pm 0.03$ & & MS-RI & \\
\hline 30.62 & 1130 & 1133 & cosmene & & & & & & & & & & & MS-RI & [46] \\
\hline 30.63 & 1168 & 1161 & Trans-p-mentha-1(7),8-dien-2-ol & $0.53 \pm 0.02$ & $1.00 \pm 0.04$ & $0.30 \pm 0.01$ & $0.48 \pm 0.03$ & & & & & & & MS-RI & \\
\hline 30.70 & 1177 & 1177 & terpinen-4-ol & $1.27 \pm 0.06$ & $1.87 \pm 0.09$ & $0.63 \pm 0.03$ & $1.09 \pm 0.10$ & & $1.02 \pm 0.07$ & & $1.23 \pm 0.09$ & $0.48 \pm 0.06$ & & Std & \\
\hline 30.98 & 1180 & 1180 & $m$-cymen-8-ol & & & & & & & & & & & Std & \\
\hline 31.15 & 1183 & 1181 & $p$-cymen-8-ol & & $0.67 \pm 0.07$ & & & & & & & $0.41 \pm 0.05$ & & Std & \\
\hline 31.32 & 1189 & 1190 & $\alpha$-terpineol & $11.22 \pm 0.51$ & $23.21 \pm 0.48$ & $4.95 \pm 0.54$ & $9.76 \pm 0.81$ & $2.82 \pm 0.18$ & $11.02 \pm 0.65$ & $7.90 \pm 0.18$ & $5.29 \pm 0.22$ & $3.05 \pm 0.18$ & $0.72 \pm 0.03$ & Std & \\
\hline 31.64 & 1192 & 1191 & estragole & $0.66 \pm 0.03$ & $0.85 \pm 0.02$ & $0.46 \pm 0.06$ & $0.97 \pm 0.09$ & & $0.94 \pm 0.09$ & & & & & MS-RI & \\
\hline 32.69 & 1217 & 1213 & Trans-carveol & & & & & & & & & & $0.04 \pm 0.01$ & MS-RI & \\
\hline 33.07 & 1230 & 1229 & nerol & & & & & & & & & & & Std & \\
\hline 33.21 & 1245 & 1242 & (2-Z)-3-hexenyl isovalerate & & & & & & & & & & & MS-RI & \\
\hline 34.01 & 1246 & 1248 & carvone & & & & & & & & & & & MS-RI & \\
\hline 34.28 & 1253 & 1255 & geraniol & & $1.22 \pm 0.03$ & & & & & & & & $0.61 \pm 0.03$ & Std & \\
\hline 34.32 & 1257 & 1256 & linalyl acetate & & & & & & & & & & & Std & \\
\hline 34.67 & 1267 & 1257 & geranial & $1.12 \pm 0.07$ & & $0.37 \pm 0.04$ & $0.83 \pm 0.07$ & & $2.97 \pm 0.12$ & $5.45 \pm 0.15$ & $2.73 \pm 0.13$ & & & Std & \\
\hline 35.92 & 1289 & 1290 & bornyl acetate & & & & & & & & & & & Std & \\
\hline 36.37 & 1299 & 1312 & carvacrol & & & & & & & & & & & Std & \\
\hline 34.25 & 1325 & 1322 & methyl geraniate & & $1.38 \pm 0.03$ & & & $0.74 \pm 0.09$ & & & & $0.77 \pm 0.09$ & $0.07 \pm 0.01$ & Std & \\
\hline 37.45 & 1326 & 1326 & 6-isoprenyl-3-methoxymethoxy-3-methyl-ciclohexene (isomer) y & & $0.63 \pm 0.02$ & & & $0.88 \pm 0.15$ & & & & $0.51 \pm 0.08$ & $0.03 \pm 0.01$ & MS & \\
\hline 37.60 & 1326 & 132 & 6-isoprenyl-3-methoxymethoxy-3-methyl-ciclohexene (isomer) y & & & & & & & & & $0.98 \pm 0.11$ & & MS & \\
\hline 37.70 & 1326 & 1326 & 6-isoprenyl-3-methoxymethoxy-3-methyl-ciclohexene (isomer) y & & & & & & & & & & & MS & \\
\hline 37.70 & 1332 & 1327 & myrten & $0.68 \pm 0.04$ & $1.40 \pm 0.04$ & & $0.54 \pm 0.02$ & & & & & & $0.05 \pm 0.01$ & MS-RI & \\
\hline 38.14 & 1349 & 13 & $\alpha$-terpi & $19.97 \pm 0.32$ & & $8.57 \pm 0.17$ & $23.56 \pm 1.25$ & & $11.15 \pm 0.23$ & $7.76 \pm 0.20$ & $3.92 \pm 0.28$ & & $0.05 \pm 0.01$ & MS-RI & \\
\hline 38.41 & 1362 & 13 & neryl ace & & & & $0.41 \pm 0.02$ & & $1.38 \pm$ & & $0.50 \pm 0.08$ & & $0.06 \pm 0.01$ & Std & \\
\hline 38.79 & 1381 & 13 & geranyl acetate & $12.30 \pm 0.66$ & $17.67 \pm 0.59$ & $5.46 \pm 0.12$ & $16.95 \pm 1.03$ & $0.99 \pm 0.16$ & $16.81 \pm 1.01$ & $9.61 \pm 0.25$ & $12.21 \pm 0.94$ & $1.00 \pm 0.04$ & $4.03 \pm 0.08$ & Std & \\
\hline 39.60 & 1388 & 13 & $\beta$-cube & & & & & & & & $1.32 \pm 0.11$ & & $0.01 \pm 0.01$ & Std & \\
\hline 39.46 & 1391 & 1395 & $\beta$-elemene & & & & $0.27 \pm 0.02$ & & & & & & $0.07 \pm 0.02$ & Std & \\
\hline $\begin{array}{l}40.10 \\
40.25\end{array}$ & 1404 & 1401 & methyleugenol & $4.52 \pm 0.27$ & $4.52 \pm 0.12$ & $1.93 \pm 0.09$ & $5.03 \pm 0.13$ & $0.66 \pm 0.12$ & $7.29 \pm 0.45$ & $3.00 \pm 0.12$ & $4.02 \pm 0.32$ & $\begin{array}{l}0.73 \pm 0.03 \\
0.32 \pm 0.03\end{array}$ & $6.74 \pm 0.12$ & MS-RI & \\
\hline $\begin{array}{l}40.25 \\
40.69\end{array}$ & 1428 & 1430 & $\begin{array}{l}\text { unknown } 1 \\
\beta \text {-caryophyllene }\end{array}$ & $1.87 \pm 0.01$ & $5.55 \pm 0.15$ & $0.79 \pm 0.07$ & $2.13 \pm 0.06$ & $0.57 \pm 0.11$ & $3.99 \pm 0.29$ & $5.12 \pm 0.42$ & $5.97 \pm 0.41$ & $\begin{array}{l}0.32 \pm 0.03 \\
0.66 \pm 0.05\end{array}$ & $0.96 \pm 0.04$ & Std & \\
\hline 40.69 & 14 & 14 & Trans- $\alpha$-bergamotene & & & & & & & & & & & MS-RI & \\
\hline 41.19 & 14 & 14 & $\gamma$-elen & & & & & & & & & & & Std & \\
\hline $\begin{array}{l}41.47 \\
41.82\end{array}$ & 1441 & 1443 & aromadendrene & & & & & & & & & & $0.16 \pm 0.01$ & Std & \\
\hline 41.82 & & & & & & & $0.33 \pm 0.06$ & & & & & & & & \\
\hline
\end{tabular}


Table 1. Cont.

\begin{tabular}{|c|c|c|c|c|c|c|c|c|c|c|c|c|c|c|c|}
\hline & & & Genotype Number & 1 & 2 & 3 & 4 & 5 & 6 & 7 & 8 & 9 & 10 & $\mathrm{ID}^{\mathrm{z}}$ & Ref. \\
\hline & & & Selection & RUM6 & RUM14 & RUB3 & RUB95 & V8 & RUM3 & RUM4 & RUM4B & RUM10 & RUM12 & & \\
\hline Rt & $\begin{array}{c}\text { KI } \\
\text { Apolar } \\
\text { Lit. }\end{array}$ & $\begin{array}{c}\mathrm{KI} \\
\text { apolar }\end{array}$ & Compound & $\%$ & $\%$ & $\%$ & $\%$ & $\%$ & $\%$ & $\%$ & $\%$ & $\%$ & $\%$ & & \\
\hline 42.21 & 1455 & 1456 & $\alpha$-humulene & $2.37 \pm 0.03$ & $7.03 \pm 0.18$ & $1.27 \pm 0.05$ & $2.95 \pm 0.17$ & & $1.71 \pm 0.09$ & $2.28 \pm 0.10$ & $1.83 \pm 0.09$ & $0.18 \pm 0.01$ & $1.92 \pm 0.14$ & Std & \\
\hline 42.43 & 1457 & 1456 & (E)- $\beta$-farnesene & & & & & & & & & & & MS-RI & \\
\hline 42.53 & 1457 & 1454 & $\alpha$-patchoulene & & & & & & & & & & & MS-RI & \\
\hline 43.04 & 1458 & 1458 & phenethyl pivalate & & & & & & & & & & & MS-RI & \\
\hline 43.12 & & & unknown 3 & & & & & & & & & & $0.24 \pm 0.01$ & & \\
\hline 42.93 & 1460 & 1460 & (Z)-methyl isoeugenol & & & & & & & & & & & MS-RI & \\
\hline 43.22 & 1460 & 1458 & alloaromadendrene & $1.81 \pm 0.14$ & $0.84 \pm 0.06$ & $0.62 \pm 0.03$ & $2.25 \pm 0.12$ & $0.71 \pm 0.04$ & & & & $1.06 \pm 0.05$ & $1.87 \pm 0.11$ & MS-RI & \\
\hline 43.33 & 1489 & 1490 & $\beta$-selinene & & & & & & & & & & & Std & \\
\hline 43.51 & 1492 & 1492 & $\gamma$-selinene & & & & & & & & & & & Std & \\
\hline 43.55 & 1498 & 1499 & $\alpha$-selinene & $1.43 \pm 0.07$ & $0.59 \pm 0.05$ & $0.40 \pm 0.03$ & $1.94 \pm 0.14$ & $0.63 \pm 0.03$ & & & & $0.86 \pm 0.04$ & $2.39 \pm 0.15$ & Std & \\
\hline 43.61 & 1500 & 1502 & bicyclogermacrene & $0.46 \pm 0.02$ & $1.02 \pm 0.08$ & $0.39 \pm 0.02$ & $0.65 \pm 0.03$ & & & & & & & Std & \\
\hline 43.68 & 1506 & 1512 & $\beta$-bisabolene & & & & & & & & & & & MS-RI & \\
\hline 44.06 & 1521 & 1520 & dihydroeugenyl butanoate & & & & & & $0.70 \pm 0.03$ & $0.25 \pm 0.01$ & $0.92 \pm 0.08$ & $0.11 \pm 0.01$ & $3.65 \pm 0.20$ & MS-RI & [13] \\
\hline 44.23 & 1523 & 1520 & $\delta$-cadinene & & & & & & & & $1.43 \pm 0.07$ & & $0.39 \pm 0.02$ & Std & \\
\hline 44.70 & 1530 & 1532 & zonarene & & & & & & & & $0.62 \pm 0.03$ & & & MS-RI & \\
\hline 44.87 & 1546 & 1543 & $\alpha$-calacorene & & & & & & & & & & $0.33 \pm 0.01$ & MS-RI & \\
\hline 44.92 & 1547 & 1546 & selina-3,7(11)-diene & & & & & & & & & & & MS-RI & \\
\hline 45.12 & 1548 & 1548 & (Z)-nerolidol & & & & & & & & $0.49 \pm 0.03$ & & $0.70 \pm 0.02$ & Std & \\
\hline 45.37 & 1550 & 1549 & elemol & & & & & & & & $1.36 \pm 0.07$ & & $0.41 \pm 0.01$ & MS-RI & \\
\hline 45.41 & 1551 & 1553 & ledol & & & & & & & & & & & MS-RI & \\
\hline 45.89 & 1578 & 1579 & spathulenol & $0.36 \pm 0.02$ & & & $0.51 \pm 0.02$ & & & & $0.35 \pm 0.01$ & & $2.77 \pm 0.19$ & MS-RI & \\
\hline 45.97 & 1580 & 1581 & Cis-caryophyllene oxide & & & & & & & & & & & MS-RI & \\
\hline 45.97 & 1583 & 1583 & Trans-caryophyllene oxide & $3.76 \pm 0.03$ & $5.63 \pm 0.28$ & $0.54 \pm 0.04$ & $2.72 \pm 0.09$ & $1.30 \pm 0.07$ & $3.03 \pm 0.12$ & $1.75 \pm 0.03$ & $4.75 \pm 0.09$ & $2.09 \pm 0.13$ & $14.73 \pm 0.25$ & MS-RI & \\
\hline 46.30 & 1594 & 1595 & Cis-arteannuic alcohol & & & & & & & & & & & MS-RI & [47] \\
\hline 46.31 & 1598 & 1596 & carotol & & & & & & & & & & & MS-RI & {$[44]$} \\
\hline 46.32 & 1601 & 1600 & guaiol & & $0.59 \pm 0.05$ & & $0.34 \pm 0.02$ & & & & & & $0.56 \pm 0.02$ & Std & \\
\hline 46.18 & 1603 & 1603 & $\alpha$-dihydro $(10,11)$ bi sabolol & & & & & & & & & & & MS-RI & \\
\hline 46.48 & 1606 & 1608 & humulene epoxide II & $2.43 \pm 0.15$ & $4.46 \pm 0.18$ & $0.73 \pm 0.04$ & $3.01 \pm 0.15$ & $0.32 \pm 0.02$ & $1.40 \pm 0.07$ & & $0.82 \pm 0.04$ & $0.78 \pm 0.03$ & & MS-RI & {$[48]$} \\
\hline 46.80 & 1613 & 1617 & isoleptospermone & & & & & & & & & & $2.36 \pm 0.09$ & MS-RI & \\
\hline $\begin{array}{l}46.82 \\
46.92\end{array}$ & 1620 & 1623 & $\begin{array}{l}\text { leptospermone } \\
\text { unknown } 4\end{array}$ & $1.33 \pm 0.08$ & $2.14 \pm 0.11$ & $0.64 \pm 0.03$ & $1.78 \pm 0.09$ & $0.62 \pm 0.04$ & & & & & $3.78 \pm 0.12$ & MS-RI & {$[49]$} \\
\hline $\begin{array}{l}40.92 \\
46.98 \\
47.06\end{array}$ & 1631 & 1631 & $\begin{array}{l}\text { unknown } 4 \\
\text { dihydroeugenyl pentanoate } \\
\text { unknown } 5\end{array}$ & 1.0010 .000 & $2.17+0.11$ & $0.07+0.00$ & 1.60 - & $0.02+0.07$ & $2.15 \pm 0.10$ & $0.97 \pm 0.06$ & $1.75 \pm 0.08$ & $1.26 \pm 0.07$ & $6.79 \pm 0.18$ & MS-RI & [13] \\
\hline $\begin{array}{l}47.06 \\
47.15\end{array}$ & 1632 & 1634 & $\gamma$-eudesmol & & & & & & & & $0.63 \pm 0.03$ & $0.37 \pm 0.01$ & $1.04 \pm 0.05$ & Std & \\
\hline 47.23 & & & Unknwon 6 & $0.65 \pm 0.03$ & $1.57 \pm 0.07$ & $0.21 \pm 0.01$ & $0.89 \pm 0.04$ & & & & & & & & \\
\hline $\begin{array}{l}47.32 \\
47.42\end{array}$ & 1641 & 1643 & $\begin{array}{l}\text { alloaromadendrene epoxide } \\
\text { unknown } 7\end{array}$ & & $0.58 \pm 0.06$ & & $0.38 \pm 0.03$ & & & & & $0.53 \pm 0.02$ & $2.74 \pm 0.18$ & MS-RI & \\
\hline 47.43 & 1641 & 1641 & $\begin{array}{l}\text { unknown } \\
5, \alpha \text { caryophylla-4(14),8(15)-dien-5-ol }\end{array}$ & & & & & & & & & & $2.47 \pm 0.17$ & MS-RI & \\
\hline 47.55 & 1642 & 1642 & $\begin{array}{l}\text { epi- } \alpha \text {-muurolol } \\
\text { ent }-1,0)\end{array}$ & & & & & & & & & & $0.51 \pm 0.02$ & MS-RI & \\
\hline 47.83 & 1644 & 1644 & $\alpha$-selinen-3,11-en-6-ol & $0.60 \pm 0.04$ & & & $0.71 \pm 0.03$ & & & & $0.45 \pm 0.02$ & & $0.55 \pm 0.02$ & MS & \\
\hline 47.89 & 1660 & 1660 & $\alpha$-selinen-11-en-4-ol & & & & & & $0.42 \pm 0.02$ & & & $0.43 \pm 0.03$ & $3.23 \pm 0.09$ & MS & \\
\hline 48.05 & 1663 & 1661 & epi-globulol & & & & & & & & & & & MS-RI & \\
\hline 47.97 & 1675 & 1670 & $\beta$-bisabolol & & & & & & & & & & $1.51 \pm 0.05$ & Std & \\
\hline 48.34 & 1682 & 1682 & ledene oxide II & & & & & & & & & & $0.46 \pm 0.03$ & MS-RI & {$[50]$} \\
\hline
\end{tabular}


Table 1. Cont.

\begin{tabular}{|c|c|c|c|c|c|c|c|c|c|c|c|c|c|c|c|}
\hline & & & Genotype Number & 1 & 2 & 3 & 4 & 5 & 6 & 7 & 8 & 9 & 10 & $\mathrm{ID}^{\mathrm{z}}$ & Ref. \\
\hline & & & Selection & RUM6 & RUM14 & RUB3 & RUB95 & $\mathrm{V} 8$ & RUM3 & RUM4 & RUM4B & RUM10 & RUM12 & & \\
\hline $\mathbf{R t}$ & $\begin{array}{c}\text { KI } \\
\text { Apolar } \\
\text { Lit. }\end{array}$ & $\underset{\text { apolar }}{\text { KI }}$ & Compound & $\%$ & $\%$ & $\%$ & $\%$ & $\%$ & $\%$ & $\%$ & $\%$ & $\%$ & $\%$ & & \\
\hline $\begin{array}{l}48.56 \\
49.22\end{array}$ & 1700 & 1713 & $\begin{array}{l}\text { eudesm-7(11)-en-4-ol } \\
\text { unknown } 8\end{array}$ & & & & & & & & & $0.63+0.04$ & $0.29 \pm 0.01$ & MS-RI & [51] \\
\hline 50.59 & 1725 & 1738 & $\begin{array}{l}\text { unknown } 8 \\
\alpha \text {-farnesol }\end{array}$ & & & & & & & & & & $0.20 \pm 0.01$ & Std & \\
\hline \multirow{3}{*}{54.25} & 1972 & 1978 & $n$-hexadecanoic acid & & & & & & & & & & & MS-RI & \\
\hline & 2000 & 1999 & eicosane & & & & & & & & & & & MS-RI & \\
\hline & & & Number of identified compounds & 26 & 24 & 25 & 29 & 18 & 21 & 17 & 30 & 29 & 40 & & \\
\hline
\end{tabular}

z ID = Identification methods. MS: by comparison of the Mass spectrum with those of the computer mass libraries Adams, Nist 11 and by interpretation of the mass spectra fragmentations. RI: by comparison of retention index with those reported in literature [8,37]. Std: by comparison of the retention time and mass spectrum of available authentic standards. MS: identification of Mass spectrum. No-polar column ZB-5. Data are the mean of three replicates \pm standard deviation. ${ }^{\mathrm{y}}$ Tentatively identified.

Table 2. Chemical composition of berry essential oil of ten (n. 11-20) myrtle genotypes.

\begin{tabular}{|c|c|c|c|c|c|c|c|c|c|c|c|c|c|c|c|}
\hline & & & $\begin{array}{c}\text { Genotype Number } \\
\text { Selection }\end{array}$ & $\begin{array}{c}11 \\
\text { RUM20 } \\
\end{array}$ & $\begin{array}{l}12 \\
\text { CPT3 }\end{array}$ & $\begin{array}{c}13 \\
\text { CPT4 } \\
\end{array}$ & $\begin{array}{l}14 \\
\text { CPT6 }\end{array}$ & $\begin{array}{l}15 \\
\text { V4 } \\
\end{array}$ & $\begin{array}{l}16 \\
\text { V5 } \\
\end{array}$ & $\begin{array}{l}17 \\
\text { V7 } \\
\end{array}$ & $\begin{array}{l}18 \\
\text { V9 } \\
\end{array}$ & $\begin{array}{c}19 \\
\text { V11 } \\
\end{array}$ & $\begin{array}{c}20 \\
\text { V12 } \\
\end{array}$ & $\mathrm{ID}^{\mathrm{z}}$ & Ref. \\
\hline Rt & $\begin{array}{c}\text { KI } \\
\text { apolar } \\
\text { it. }\end{array}$ & $\begin{array}{c}\text { KI } \\
\text { apolar }\end{array}$ & Compound & $\%$ & $\%$ & $\%$ & $\%$ & $\%$ & $\%$ & $\%$ & $\%$ & $\%$ & $\%$ & & \\
\hline 17.88 & 939 & 937 & $\alpha$-pinene & & & & $3.18 \pm 0.06$ & $7.02 \pm 0.36$ & & & & & & Std & \\
\hline 21.40 & 986 & 985 & butanoic acid, 2-methyl-2-methylpropyl ester & & & & & & & & & & & MS-RI & [43] \\
\hline 21.6 & 1002 & 1001 & $\alpha$-phellandrene & & & & $0.33 \pm 0.03$ & & & & & & & Std & \\
\hline 21.93 & 1002 & 1002 & $\delta$-2-carene & & & & $4.53 \pm 0.38$ & $1.19 \pm 0.06$ & & & & & & Std & \\
\hline 22.25 & 1005 & 1004 & pseudolimonene & & & & $0.22 \pm 0.02$ & & & & & & & MS-RI & [44] \\
\hline 22.66 & 1025 & 1021 & $p$-cymene & & & & $12.83 \pm 0.68$ & $3.31 \pm 0.04$ & & & & & $1.04 \pm 0.09$ & Std & \\
\hline 22.90 & 1031 & 1029 & limonene & & & & $10.22 \pm 0.55$ & $5.99 \pm 0.18$ & & & & & $4.58 \pm 0.28$ & Std & \\
\hline 23.07 & 1035 & 1031 & 1,8-cineole & & & & $18.72 \pm 0.37$ & $43.26 \pm 1.96$ & & & $0.13 \pm 0.01$ & & $3.79 \pm 0.19$ & Std & \\
\hline 24.49 & 1060 & 1057 & $\gamma$-terpinene & & & & $11.37 \pm 0.26$ & $1.78 \pm 0.08$ & & & & & & Std & \\
\hline 26.08 & 1089 & 1063 & $\alpha$-terpinolene & & & & $5.69 \pm 0.14$ & $0.77 \pm 0.05$ & & & & & & Std & \\
\hline 26.55 & 1097 & 1094 & linalool & $1.01 \pm 0.07$ & & $0.60 \pm 0.03$ & $9.94 \pm 0.54$ & $3.68 \pm 0.17$ & $1.61 \pm 0.07$ & & $2.40 \pm 0.19$ & $2.77 \pm 0.21$ & $4.49 \pm 0.21$ & Std & \\
\hline 26.75 & 1112 & 1108 & n-amyl isovalerate & & & & $2.42 \pm 0.08$ & $3.07 \pm 0.18$ & & & $1.50 \pm 0.12$ & & $2.07 \pm 0.11$ & MS-RI & [45] \\
\hline 28.81 & 1113 & 1115 & Trans-pinocarveol & & & & $0.25 \pm 0.02$ & & & & $0.51 \pm 0.03$ & & $0.42 \pm 0.03$ & MS-RI & \\
\hline 30.62 & 1130 & 1133 & cosmene & & & & & & & & & $0.74 \pm 0.09$ & $0.47 \pm 0.03$ & MS-RI & [46] \\
\hline 30.63 & 1168 & 1161 & Trans-p-mentha-1(7),8-dien-2-ol & & & & $0.87 \pm 0.11$ & & & & & & & MS-RI & \\
\hline 30.70 & 1177 & 1177 & terpinen-4-ol & $0.69 \pm 0.03$ & & $0.42 \pm 0.02$ & $2.46 \pm 0.15$ & $0.69 \pm 0.09$ & & & & $1.38 \pm 0.07$ & $1.50 \pm 0.04$ & Std & \\
\hline 30.98 & 1180 & 1180 & $m$-cymen-8-ol & & & & & & & & & & & Std & \\
\hline 31.15 & 1183 & 1181 & $p$-cymen-8-ol & & & & $0.72 \pm 0.06$ & & & & $0.57 \pm 0.03$ & $10.16 \pm 0.51$ & $0.39 \pm 0.03$ & Std & \\
\hline 31.32 & 1189 & 1190 & $\alpha$-terpineol & $8.23 \pm 0.43$ & $1.81 \pm 0.09$ & $4.90 \pm 0.24$ & $6.12 \pm 0.23$ & $6.82 \pm 0.87$ & & & $9.15 \pm 0.46$ & & $11.51 \pm 0.69$ & Std & \\
\hline 31.64 & 1192 & 1191 & estragole & & & & & & & & $0.77 \pm 0.04$ & $0.66 \pm 0.03$ & $0.66 \pm 0.03$ & MS-RI & \\
\hline 32.69 & 1217 & 1213 & Trans-carveol & & & & & & & & $0.42 \pm 0.02$ & & $0.36 \pm 0.02$ & MS-RI & \\
\hline 33.07 & 1230 & 1229 & nerol & & & & & & & & & & & Std & \\
\hline 33.21 & 1245 & 1242 & (2-Z)-3-hexenyl isovalerate & & & & $0.34 \pm 0.02$ & & & & $0.58 \pm 0.03$ & & & MS-RI & \\
\hline 34.01 & 1246 & 1248 & carvone & & & & & & & & & & & MS-RI & \\
\hline 34.28 & 1253 & 1255 & geraniol & & & & $0.20 \pm 0.01$ & & & & & & & Std & \\
\hline 34.32 & 1257 & 1256 & linalyl acetate & & & & & & & & & & & Std & \\
\hline
\end{tabular}


Table 2. Cont

\begin{tabular}{|c|c|c|c|c|c|c|c|c|c|c|c|c|c|c|c|}
\hline & & & $\begin{array}{l}\text { Genotype Number } \\
\text { Selection }\end{array}$ & $\begin{array}{c}11 \\
\text { RUM20 }\end{array}$ & $\begin{array}{l}12 \\
\text { CPT3 }\end{array}$ & $\begin{array}{c}13 \\
\text { CPT4 }\end{array}$ & $\begin{array}{l}14 \\
\text { CPT6 }\end{array}$ & $\begin{array}{l}15 \\
\text { V4 }\end{array}$ & $\begin{array}{l}16 \\
\text { V5 }\end{array}$ & $\begin{array}{l}17 \\
\text { V7 }\end{array}$ & $\begin{array}{l}18 \\
\text { V9 }\end{array}$ & $\begin{array}{c}19 \\
\text { V11 }\end{array}$ & $\begin{array}{c}20 \\
\text { V12 }\end{array}$ & $\mathrm{ID}^{\mathrm{z}}$ & Ref. \\
\hline Rt & $\begin{array}{c}\text { KI } \\
\text { apolar } \\
\text { Lit. }\end{array}$ & $\begin{array}{c}\mathrm{KI} \\
\text { apolar }\end{array}$ & Compound & $\%$ & $\%$ & $\%$ & $\%$ & $\%$ & $\%$ & $\%$ & $\%$ & $\%$ & $\%$ & & \\
\hline 34.67 & 1267 & 1257 & geranial & & $0.64 \pm 0.03$ & $2.48 \pm 0.18$ & & $1.34 \pm 0.04$ & $4.68 \pm 0.22$ & & $0.77 \pm 0.06$ & $1.44 \pm 0.07$ & $1.82 \pm 0.08$ & Std & \\
\hline 35.92 & 1289 & 1290 & bornyl acetate & & & & & & & & & & & Std & \\
\hline 36.37 & 1299 & 1312 & carvacrol & & & & & & & & & & & Std & \\
\hline 34.25 & 1325 & 1322 & methyl geraniate & $0.98 \pm 0.06$ & & & & & & & $0.45 \pm 0.02$ & $0.87 \pm 0.06$ & $0.50 \pm 0.04$ & Std & \\
\hline 37.45 & 1326 & 1326 & 6-isoprenyl-3-methoxymethoxy-3-methyl-ciclohexene (isomer) y & & & & & & & & $0.21 \pm 0.01$ & & $0.41 \pm 0.03$ & MS & \\
\hline 37.60 & 1326 & 1326 & 6-isoprenyl-3-methoxymethoxy-3-methyl-ciclohexene (isomer) y & $1.04 \pm 0.08$ & & $0.57 \pm 0.04$ & & & $0.46 \pm 0.02$ & & $0.63 \pm 0.03$ & & $1.45 \pm 0.09$ & MS & \\
\hline 37.70 & 1326 & 1326 & 6-isoprenyl-3-methoxymethoxy-3-methyl-ciclohexene (isomer) y & & & & & & & & & & & MS & \\
\hline 37.70 & 1332 & 1327 & myrtenyl acetate & $1.11 \pm 0.09$ & & $0.84 \pm 0.05$ & & & & & & $0.81 \pm 0.05$ & $0.73 \pm 0.05$ & MS-RI & \\
\hline 38.14 & 1349 & 1351 & $\alpha$-terpinyl acetate & $12.43 \pm 0.73$ & $6.70 \pm 0.13$ & $20.24 \pm 0.92$ & & $6.51 \pm 0.39$ & $14.4 \pm 1.04$ & & $7.9 \pm 0.43$ & $18.56 \pm 1.26$ & $13.3 \pm 0.93$ & MS-RI & \\
\hline 38.41 & 1362 & 1366 & neryl acetate & & $16.54 \pm 0.71$ & $1.21 \pm 0.05$ & $4.14 \pm 0.11$ & & $2.84 \pm 0.16$ & & $0.47 \pm 0.02$ & $0.58 \pm 0.04$ & $0.78 \pm 0.06$ & Std & \\
\hline 38.79 & 1381 & 1379 & geranyl acetate & $21.38 \pm 1.66$ & & $43.27 \pm 1.98$ & & $8.57 \pm 0.46$ & $50.95 \pm 2.58$ & & $27.78 \pm 2.01$ & $16.79 \pm 1.04$ & $21.61 \pm 1.82$ & Std & \\
\hline 39.60 & 1388 & 1388 & $\beta$-cubebene & & & & & & & & & & & Std & \\
\hline 39.46 & 1391 & 1395 & $\beta$-elemene & & $1.34 \pm 0.07$ & $1.15 \pm 0.05$ & & & & & & & & Std & \\
\hline 40.10 & 1404 & 1401 & methyleugenol & $12.32 \pm 0.80$ & $1.49 \pm 0.08$ & $12.16 \pm 0.47$ & $0.58 \pm 0.02$ & $2.68 \pm 0.11$ & $1.11 \pm 0.05$ & & $12.34 \pm 0.31$ & $6.82 \pm 0.38$ & $7.28 \pm 0.81$ & MS-RI & \\
\hline $\begin{array}{l}40.69 \\
40.69\end{array}-x-3$ & 1428 & 1430 & $\begin{array}{l}\text { unnown } 1 \\
\beta \text {-caryophyllene }\end{array}$ & & & & & & & & & & & Std & \\
\hline 40.69 & 1435 & 1439 & Trans- $\alpha$-bergamotene & & & & & & & & & & & MS-RI & \\
\hline 41.19 & 1437 & 1434 & $\gamma$-elemene & & & & & & & & & & & Std & \\
\hline 41.47 & 1441 & 1443 & aromadendrene & & $1.37 \pm 0.09$ & & & & & & $0.16 \pm 0.01$ & & & Std & \\
\hline 41.82 & & & unknown 2 & & & & & & & & & & & Std & \\
\hline 42.21 & 1455 & 1456 & $\alpha$-humulene & $11.98 \pm 0.78$ & $18.92 \pm 1.09$ & $0.93 \pm 0.02$ & $0.72 \pm 0.03$ & $1.6 \pm 0.08$ & $2.48 \pm 0.12$ & $23.75 \pm 1.87$ & $3.11 \pm 0.54$ & $6.82 \pm 0.41$ & $3.14 \pm 0.65$ & Std & \\
\hline 42.43 & 1457 & 1456 & (E)- $\beta$-farnesene & & $0.99 \pm 0.04$ & & & & & & & & & MS-RI & \\
\hline 42.53 & 1457 & 1454 & $\alpha$-patchoulene & & $0.71 \pm 0.03$ & & & & & & & & & MS-RI & \\
\hline 43.04 & 1458 & 1458 & phenethyl pivalate & & & $0.34 \pm 0.01$ & & & $1.03 \pm 0.05$ & & & & & MS-RI & \\
\hline $\begin{array}{l}43.12 \\
42.93\end{array}$ & 1460 & 1460 & $\begin{array}{l}\text { unknown } 3 \\
\text { (Z)-methyl isoeugenol }\end{array}$ & & & & & & & & $0.15 \pm 0.01$ & & & MS-RI & \\
\hline 43.22 & 1460 & 1458 & $\begin{array}{l}\text { alloaromadendrene } \\
\text { a d }\end{array}$ & & $8.17 \pm 0.11$ & $1.46 \pm 0.04$ & & & & & $0.22 \pm 0.01$ & $0.62 \pm 0.04$ & & MS-RI & \\
\hline 43.33 & 1489 & 1490 & $\beta$-selinene & & & & & & & & & & & Std & \\
\hline 43.51 & 1492 & 1492 & $\gamma$-selinene & & & & & & & & & & & Std & \\
\hline 43.55 & 1498 & 1499 & $\alpha$-selinene & & $8.94 \pm 0.12$ & $1.36 \pm 0.03$ & & & & & $0.13 \pm 0.01$ & & & Std & \\
\hline 43.61 & 1500 & 1502 & bicyclogermacrene & & & & & & & & & & & Std & \\
\hline 43.68 & 1506 & 1512 & $\beta$-bisabolene & & & & & & & & & & & MS-RI & \\
\hline 44.06 & 1521 & 1520 & dihydroeugenyl butanoate & $1.33 \pm 0.06$ & $0.10 \pm 0.01$ & & $0.30 \pm 0.02$ & & $0.43 \pm 0.03$ & $7.82 \pm 0.65$ & $2.52 \pm 0.15$ & $0.85 \pm 0.05$ & $0.93 \pm 0.08$ & MS-RI & [13] \\
\hline 44.23 & 1523 & 1520 & $\delta$-cadinene & & & & & & & & & & & Std & \\
\hline 44.70 & 1530 & 1532 & zonarene & & & & $0.15 \pm 0.01$ & & & & $0.18 \pm 0.01$ & & & MS-RI & \\
\hline 44.87 & 15 & 1543 & $\alpha$-calacorene & & & & & & & & & & & MS-RI & \\
\hline 44.92 & 154 & 1546 & selina-3,7(11)-diene & & & & & & & & & & & MS-RI & \\
\hline 45.1 & 15 & 1548 & (Z)-nerolidol & & & & & & & & & & & Std & \\
\hline 45.3 & 15 & 1549 & elemol & $0.66 \pm 0.04$ & & & $0.28 \pm 0.01$ & & & & & & & MS-RI & \\
\hline 45.41 & 1551 & 1553 & ledol & & & & & & & & & & & MS-RI & \\
\hline $\begin{array}{r}45.89 \\
45.97\end{array}$ & 1578 & 1579 & spathulenol & & $1.05 \pm 0.05$ & $1.94 \pm 0.05$ & & & & & $0.26 \pm 0.01$ & & & MS-RI & \\
\hline 45.97 & 1580 & 1581 & Cis-caryophyllene oxide & & & & & & & & & & & MS-RI & \\
\hline
\end{tabular}


Table 2. Cont.

\begin{tabular}{|c|c|c|c|c|c|c|c|c|c|c|c|c|c|c|c|}
\hline & & & $\begin{array}{l}\text { Genotype Number } \\
\text { Selection }\end{array}$ & $\begin{array}{c}11 \\
\text { RUM20 }\end{array}$ & $\begin{array}{l}12 \\
\text { CPT3 }\end{array}$ & $\begin{array}{l}13 \\
\text { CPT4 }\end{array}$ & $\begin{array}{l}14 \\
\text { CPT6 }\end{array}$ & $\begin{array}{l}15 \\
\text { V4 }\end{array}$ & $\begin{array}{l}16 \\
\text { V5 }\end{array}$ & $\begin{array}{l}17 \\
\text { V7 }\end{array}$ & $\begin{array}{l}18 \\
\text { V9 }\end{array}$ & $\begin{array}{c}19 \\
\text { V11 }\end{array}$ & $\begin{array}{c}20 \\
\text { V12 }\end{array}$ & $\mathrm{ID}^{\mathrm{z}}$ & Ref. \\
\hline Rt & $\begin{array}{c}\text { KI } \\
\text { apolar } \\
\text { Lit. }\end{array}$ & $\begin{array}{c}\text { KI } \\
\text { apolar }\end{array}$ & Compound & $\%$ & $\%$ & $\%$ & $\%$ & $\%$ & $\%$ & $\%$ & $\%$ & $\%$ & $\%$ & & \\
\hline 45.97 & 1583 & 1583 & Trans-caryophyllene oxide & $5.81 \pm 0.38$ & $6.39 \pm 0.35$ & $1.95 \pm 0.04$ & $1.10 \pm 0.04$ & & $8.65 \pm 0.60$ & $17.27 \pm 0.91$ & $5.52 \pm 0.12$ & $9.13 \pm 0.26$ & $3.94 \pm 0.03$ & MS-RI & \\
\hline 46.30 & 1594 & 1595 & Cis-arteannuic alcohol & $0.70 \pm 0.05$ & & & & & & & & & & MS-RI & [47] \\
\hline 46.31 & 1598 & 1596 & carotol & & & & & & $0.55 \pm 0.04$ & & & & & MS-RI & [44] \\
\hline 46.32 & 1601 & 1600 & guaiol & & $0.34 \pm 0.02$ & & & & & & & & & Std & \\
\hline 46.18 & 1603 & 1603 & $\alpha$-dihydro $(10,11)$ bi sabolol & & & & & & $0.44 \pm 0.06$ & & $0.34 \pm 0.02$ & $1.09 \pm 0.09$ & $0.35 \pm 0.02$ & MS-RI & \\
\hline 46.48 & 1606 & 1608 & humulene epoxide II & $5.80 \pm 0.39$ & $5.59 \pm 0.42$ & $0.63 \pm 0.03$ & $0.41 \pm 0.02$ & $0.55 \pm 0.02$ & $2.25 \pm 0.08$ & $13.68 \pm 0.83$ & $2.07 \pm 0.04$ & $9.62 \pm 0.75$ & $3.30 \pm 0.47$ & MS-RI & [48] \\
\hline 46.80 & 1613 & 1617 & isoleptospermone & & & & & & & & & & & MS-RI & \\
\hline $\begin{array}{l}46.82 \\
46.92\end{array}$ & 1620 & 1623 & $\begin{array}{l}\text { leptospermone } \\
\text { unknown } 4\end{array}$ & & & $0.41 \pm 0.02$ & & & & & & & & MS-RI & [49] \\
\hline $\begin{array}{l}46.98 \\
47.06\end{array}$ & 1631 & 1631 & $\begin{array}{l}\text { dihydroeugenyl pentanoate } \\
\text { unknown } 5\end{array}$ & $2.70 \pm 0.27$ & & $0.10 \pm 0.01$ & $0.15 \pm 0.01$ & $0.40 \pm 0.02$ & $1.07 \pm 0.04$ & $10.84 \pm 0.58$ & $2.52 \pm 0.18$ & $2.73 \pm 0.19$ & $3.46 \pm 0.58$ & MS-RI & [13] \\
\hline $\begin{array}{l}47.15 \\
47.23\end{array}$ & 1632 & 1634 & $\begin{array}{l}\gamma \text {-eudesmol } \\
\text { Unknwon } 6\end{array}$ & & & & & & & & $0.35 \pm 0.02$ & & & Std & \\
\hline $\begin{array}{l}47.32 \\
47.42\end{array}$ & 1641 & 1643 & $\begin{array}{l}\text { alloaromadendrene epoxide } \\
\text { unknown } 7\end{array}$ & $1.54 \pm 0.16$ & $1.13 \pm 0.06$ & & & & $0.47 \pm 0.02$ & $10.79 \pm 0.49$ & $0.75 \pm 0.03$ & $1.72 \pm 0.12$ & $0.81 \pm 0.05$ & MS-RI & \\
\hline 47.43 & 1641 & 1641 & $5, \alpha$ caryophylla-4(14),8(15)-dien-5-ol & $0.78 \pm 0.05$ & $0.38 \pm 0.02$ & & & & $0.50 \pm 0.02$ & & $0.65 \pm 0.03$ & $0.56 \pm 0.04$ & $0.41 \pm 0.02$ & MS-RI & \\
\hline 47.55 & 1642 & 1642 & epi- $\alpha$-muurolol & & & & & & & & & & & MS-RI & \\
\hline 47.83 & 1644 & 1644 & $\alpha$-selinen-3,11-en-6-ol & $0.52 \pm 0.03$ & $0.49 \pm 0.03$ & & & & & & $0.74 \pm 0.06$ & & & MS & \\
\hline 47.89 & 1660 & 1660 & $\alpha$-selinen-11-en-4 -ol & & $1.85 \pm 0.11$ & $1.75 \pm 0.05$ & & & & & $0.46 \pm 0.02$ & & & MS & \\
\hline 48.05 & 1663 & 1661 & epi-globulol & & & & & & & & & & & MS-RI & \\
\hline 47.97 & 1675 & 1670 & $\beta$-bisabolol & & & & & & & & $0.21 \pm 0.01$ & & & Std & \\
\hline 48.34 & 1682 & 1682 & ledene oxide II & & & & & & & & & & & MS-RI & [50] \\
\hline 48.56 & 1700 & 1713 & eudesm-7(11)-en-4-ol & & & & & & & & & & & MS-RI & [51] \\
\hline 49.22 & & & unknown 8 & & & & & & & & & & & & \\
\hline 50.59 & 1725 & 1738 & $\alpha$-farnesol & & & & & & & & & & & Std & \\
\hline 51.29 & 1972 & 1978 & $n$-hexadecanoic acid & & & & & & & & & & & MS-RI & \\
\hline \multirow[t]{2}{*}{54.25} & 2000 & 1999 & eicosane & & & & & & & & & & & MS-RI & \\
\hline & & & Number of identified comp & 19 & 21 & 21 & 27 & 18 & 17 & 6 & 35 & 21 & 29 & & \\
\hline
\end{tabular}

${ }^{\mathrm{z}}$ ID = Identification methods. MS: by comparison of the Mass spectrum with those of the computer mass libraries Adams, Nist 11 and by interpretation of the mass spectra fragmentations. RI: by comparison of retention index with those reported in literature [8,37]. Std: by comparison of the retention time and mass spectrum of available authentic standards. MS: identification of Mass spectrum. No-polar column ZB-5. Data are the mean of three replicates \pm standard deviation. ${ }^{\mathrm{y}}$ Tentatively identified. 
Table 3. Chemical composition of berry essential oil of ten (n. 21-30) myrtle genotypes.

\begin{tabular}{|c|c|c|c|c|c|c|c|c|c|c|c|c|c|c|c|}
\hline & & & $\begin{array}{l}\text { Genotype Number } \\
\text { Selection }\end{array}$ & $\begin{array}{c}21 \\
\text { V15 } \\
\end{array}$ & $\begin{array}{c}22 \\
\text { V16 } \\
\end{array}$ & $\begin{array}{c}23 \\
\text { V17 } \\
\end{array}$ & $\begin{array}{l}24 \\
\text { V19 } \\
\end{array}$ & $\begin{array}{c}25 \\
\text { V20 } \\
\end{array}$ & $\begin{array}{c}26 \\
\text { LAC1 }\end{array}$ & $\begin{array}{c}27 \\
\text { LAC11 } \\
\end{array}$ & $\begin{array}{c}28 \\
\text { LAC31 } \\
\end{array}$ & $\begin{array}{c}29 \\
\text { BOS1 }\end{array}$ & $\begin{array}{c}30 \\
\text { BOS2 }\end{array}$ & $\mathrm{ID}^{\mathrm{z}}$ & Ref. \\
\hline Rt & $\begin{array}{c}\text { KI } \\
\text { Appolar } \\
\text { Lit. }\end{array}$ & $\begin{array}{c}\text { KI } \\
\text { Apolar }\end{array}$ & Compound & $\%$ & $\%$ & $\%$ & $\%$ & $\%$ & $\%$ & $\%$ & $\%$ & $\%$ & $\%$ & & \\
\hline 17.88 & 939 & 937 & $\alpha$-pinene & & & & & & & & $0.35 \pm 0.02$ & $5.59 \pm 0.21$ & & Std & \\
\hline 21.40 & 986 & 985 & butanoic acid, 2-methyl-2-methylpropyl ester & & & & & & & & & & & MS-RI & [43] \\
\hline $\begin{array}{l}21.6 \\
21.93\end{array}$ & 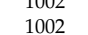 & 1002 & $\begin{array}{l}\alpha \text {-phellandrene } \\
\delta \text {-2-arene }\end{array}$ & & & & $0.23 \pm 0.02$ & & $0.19 \pm 0.01$ & & & & & $\begin{array}{l}\text { Std } \\
\text { Std }\end{array}$ & \\
\hline 22.25 & 1005 & 1004 & pseudolimonene & & & & & & & & & & & MS-RI & [44] \\
\hline 22.66 & 1025 & 1021 & p-cymene & & $2.91 \pm 0.06$ & & & $0.94 \pm 0.05$ & & & $0.66 \pm 0.04$ & $2.74 \pm 0.13$ & & Std & \\
\hline 22.90 & 1031 & 1029 & limonene & & $12.87 \pm 0.98$ & $1.21 \pm 0.04$ & & $1.19 \pm 0.07$ & & & $0.38 \pm 0.02$ & $9.08 \pm 0.45$ & & Std & \\
\hline 23.07 & 1035 & 1031 & 1,8-cineole & & $14.01 \pm 0.69$ & $2.46 \pm 0.09$ & & $2.22 \pm 0.13$ & $0.09 \pm 0.01$ & & $2.65 \pm 0.14$ & $41.18 \pm 2.10$ & & Std & \\
\hline 24.49 & 1060 & 1057 & $\gamma$-terpinene & & $2.70 \pm 0.10$ & & & $0.59 \pm 0.04$ & & $5.27 \pm 0.41$ & $0.39 \pm 0.03$ & $1.97 \pm 0.09$ & & Std & \\
\hline 26.08 & 1089 & 1063 & $\alpha$-terpinolene & & $0.95 \pm 0.07$ & & & $0.24 \pm 0.02$ & & & $0.53 \pm 0.04$ & $1.00 \pm 0.06$ & & Std & \\
\hline 26.55 & 1097 & 1094 & linalool & $2.70 \pm 0.05$ & $7.83 \pm 0.19$ & $28.71 \pm 1.24$ & $10.86 \pm 0.59$ & $34.04 \pm 2.16$ & $1.75 \pm 0.01$ & & $10.63 \pm 0.61$ & $3.46 \pm 0.14$ & $1.90 \pm 0.09$ & Std & \\
\hline 26.75 & 1112 & 1108 & n-amyl isovalerate & $0.33 \pm 0.02$ & $3.18 \pm 0.15$ & $2.18 \pm 0.09$ & $0.50 \pm 0.04$ & $1.92 \pm 0.16$ & $0.09 \pm 0.01$ & & & & & MS-RI & [45] \\
\hline 28.81 & 1113 & 1115 & Trans-pinocarveol & $0.36 \pm 0.02$ & & & & $0.35 \pm 0.04$ & $0.17 \pm 0.01$ & & & & & MS-RI & \\
\hline 30.62 & 1130 & 1133 & cosmene & & $1.29 \pm 0.07$ & $0.94 \pm 0.04$ & $0.43 \pm 0.03$ & $0.61 \pm 0.07$ & $0.60 \pm 0.05$ & $0.93 \pm 0.06$ & & & & MS-RI & [46] \\
\hline 30.63 & 1168 & 1161 & Trans-p-mentha-1(7),8-dien-2-ol & & & & & & & & & & & MS-RI & \\
\hline 30.70 & 1177 & 1177 & terpinen-4-ol & $0.74 \pm 0.04$ & $2.00 \pm 0.08$ & $1.74 \pm 0.07$ & $0.55 \pm 0.04$ & $0.90 \pm 0.06$ & $0.84 \pm 0.06$ & $2.63 \pm 0.10$ & $1.56 \pm 0.07$ & & & Std & \\
\hline 30.98 & 1180 & 1180 & m-cymen-8-ol & & & & & & & & & & & Std & \\
\hline 31.15 & 1183 & 1181 & p-cymen-8-ol & & $0.60 \pm 0.03$ & & $0.13 \pm 0.01$ & & $0.58 \pm 0.04$ & $0.47 \pm 0.03$ & & & & Std & \\
\hline 31.32 & 1189 & 1190 & $\alpha$-terpineol & $5.14 \pm 0.12$ & $12.50 \pm 0.24$ & $11.87 \pm 0.86$ & $2.65 \pm 0.10$ & $5.80 \pm 0.27$ & $68 \pm 0.17$ & $17.55 \pm 0.84$ & $5.73 \pm 0.26$ & $3.14 \pm 0.19$ & $4.68 \pm 0.20$ & Std & \\
\hline 31.64 & 1192 & 1191 & estragole & $0.30 \pm 0.02$ & $0.75 \pm 0.04$ & $0.77 \pm 0.06$ & $0.43 \pm 0.03$ & $0.35 \pm 0.04$ & $0.29 \pm 0.02$ & $0.65 \pm 0.04$ & & & & MS-RI & \\
\hline 32.69 & 1217 & 1213 & Trans-carveol & & & & & & $0.09 \pm 0.01$ & & & & & MS-RI & \\
\hline 33.07 & 1230 & 1229 & nerol & & & & $0.16 \pm 0.01$ & & & & $0.17 \pm 0.01$ & & & Std & \\
\hline 33.21 & 1245 & 1242 & (2-Z)-3-hexenyl isovalerate & $0.25 \pm 0.01$ & & & $0.22 \pm 0.01$ & & $0.15 \pm 0.01$ & & & & & $\begin{array}{l}\text { MS-RI } \\
\text { MS-RI }\end{array}$ & \\
\hline $\begin{array}{l}34.01 \\
3428\end{array}$ & $\begin{array}{l}1246 \\
1253\end{array}$ & $\begin{array}{l}1248 \\
1255\end{array}$ & $\begin{array}{l}\text { carvone } \\
\text { geraniol }\end{array}$ & $094+006$ & & & & & & & & & & MS-RI & \\
\hline 34.32 & 1257 & 1256 & $\begin{array}{l}\text { geraniol } \\
\text { linalyl acetate }\end{array}$ & $0.94 \pm 0.06$ & & & $\begin{array}{l}.52 \pm 0.03 \\
4.94 \pm 0.05\end{array}$ & & & & & & & $\begin{array}{l}\text { Std } \\
\text { Std }\end{array}$ & \\
\hline 34.67 & 1267 & 1257 & geranial & & & $2.72 \pm 0.09$ & & $0.55 \pm 0.04$ & $1.36 \pm 0.07$ & $1.75 \pm 0.06$ & $7.63 \pm 0.39$ & $1.16 \pm 0.06$ & $1.58 \pm 0.12$ & Std & \\
\hline 35.92 & 1289 & 1290 & bornyl acetate & & & & $0.12 \pm 0.01$ & & $0.12 \pm 0.01$ & $24.29 \pm 1.16$ & & & & Std & \\
\hline 36.37 & 1299 & 1312 & carvacrol & & & & $0.10 \pm 0.01$ & & $0.12 \pm 0.01$ & & & & & Std & \\
\hline 34.25 & 1325 & 1322 & methyl & $1.01 \pm 0.07$ & & & $0.30 \pm 0.02$ & $0.49 \pm 0.03$ & & & & & & Std & \\
\hline 37.45 & 1326 & 1326 & 6-isoprenyl-3-methoxymethoxy-3-m & $0.41 \pm 0.03$ & & & $0.28 \pm 0.02$ & & & & & & & MS & \\
\hline 37.60 & 1326 & 1326 & 6-isopr & $0.80 \pm 0.05$ & $1.06 \pm 0.07$ & & $0.65 \pm$ & & & & $0.22 \pm 0.01$ & & & MS & \\
\hline 37.70 & 1326 & 1326 & 3-methoxymethoxy-3-methyl-ciclohe & $1.08 \pm 0.07$ & $1.21 \pm 0.06$ & & $0.33 \pm$ & & $0.12 \pm 0.01$ & & & & & MS & \\
\hline 37.70 & 1332 & 1327 & myrter & & & $0.46 \pm 0.03$ & $0.10 \pm 0.01$ & $0.42 \pm 0.03$ & & & $0.20 \pm 0.01$ & & & MS-RI & \\
\hline 38.14 & $134 \mathrm{C}$ & 1351 & $\alpha$-terpi & & & $7.36 \pm 0.13$ & $3.35 \pm$ & & $8.45 \pm 0.66$ & & .45 & $4.21 \pm 0.22$ & $6.62 \pm 0.33$ & MS-RI & \\
\hline 38.41 & 1362 & 13 & neryl & $1 \pm 0.02$ & & $1.17=$ & 1.36 & & & & & & $15.94 \pm 0.78$ & 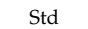 & \\
\hline 38.79 & 1381 & 1379 & geranyl & $12.56 \pm 0.85$ & $7.34 \pm 0.18$ & $17.93 \pm 0.63$ & $17.32 \pm 0.96$ & $6.88 \pm 0.37$ & $15.15 \pm 1.03$ & & $10.23 \pm 0.52$ & $8.31 \pm 0.41$ & & Std & \\
\hline 39.60 & 1388 & 1388 & $\beta$-cub & & & & & $0.42 \pm 0.03$ & & & & & & Std & \\
\hline 39.46 & 1391 & 1395 & $\beta$-ele & $0.33 \pm 0.02$ & & & & & $0.46 \pm 0.03$ & $1.46 \pm 0.07$ & & $1.54 \pm 0.08$ & $2.37 \pm 0.11$ & Std & \\
\hline 40.10 & 1404 & 1401 & met & $11.14 \pm 0.78$ & $9.88 \pm 0.52$ & $9.20 \pm 0.19$ & $6.41 \pm 0.18$ & $5.79 \pm 0.26$ & $10.03 \pm 0.55$ & $19.66 \pm 1.02$ & $3.35 \pm 0.17$ & $3.65 \pm 0.18$ & $9.17 \pm 0.56$ & MS-RI & \\
\hline $\begin{array}{l}40.25 \\
40.69\end{array}$ & 1428 & 1430 & $\begin{array}{l}\text { unknown } 1 \\
\beta \text {-caryophyllene }\end{array}$ & $6.76 \pm 0.14$ & $8.37 \pm 0.09$ & $4.47 \pm 0.08$ & $2.71 \pm 0.11$ & $5.25 \pm 0.29$ & $0.25 \pm 0.02$ & $4.24 \pm 0.23$ & $11.67 \pm 0.56$ & $5.31 \pm 0.25$ & $23.02 \pm 1.14$ & Std & \\
\hline $\begin{array}{l}40.07 \\
40.69\end{array}$ & 1435 & 1439 & Trans- $\alpha$-bergamotene & 0.010 .01 & & & $0.17 \pm 0.02$ & & & & $0.24 \pm 0.02$ & & & MS-RI & \\
\hline 41.19 & 1437 & 1434 & $\gamma$-elemene & $0.27 \pm 0.02$ & & & & & & & & & & Std & \\
\hline
\end{tabular}


Table 3. Cont.

\begin{tabular}{|c|c|c|c|c|c|c|c|c|c|c|c|c|c|c|c|}
\hline & & & $\begin{array}{c}\text { Genotype Number } \\
\text { Selection }\end{array}$ & $\begin{array}{c}21 \\
\text { V15 } \\
\end{array}$ & $\begin{array}{l}22 \\
\text { V16 } \\
\end{array}$ & $\begin{array}{c}23 \\
\text { V17 } \\
\end{array}$ & $\begin{array}{c}24 \\
\text { V19 } \\
\end{array}$ & $\begin{array}{c}25 \\
\text { V20 } \\
\end{array}$ & $\begin{array}{l}26 \\
\text { LAC1 }\end{array}$ & $\begin{array}{c}27 \\
\text { LAC11 } \\
\end{array}$ & $\begin{array}{c}28 \\
\text { LAC31 } \\
\end{array}$ & $\begin{array}{l}29 \\
\text { BOS1 }\end{array}$ & $\begin{array}{c}30 \\
\text { BOS2 }\end{array}$ & ID $^{z}$ & Ref. \\
\hline Rt & $\begin{array}{c}\text { KI } \\
\text { Apolar } \\
\text { Lit. } \\
\end{array}$ & $\begin{array}{c}\text { KI } \\
\text { Apolar }\end{array}$ & Compound & $\%$ & $\%$ & $\%$ & $\%$ & $\%$ & $\%$ & $\%$ & $\%$ & $\%$ & $\%$ & & \\
\hline $\begin{array}{l}41.47 \\
41.82\end{array}$ & 1441 & 1443 & $\begin{array}{l}\text { aromadendrene } \\
\text { unknown } 2\end{array}$ & & & & & & $0.17 \pm 0.01$ & $0.61 \pm 0.05$ & & & $1.70 \pm 0.09$ & $\begin{array}{l}\mathrm{Std} \\
\mathrm{Std}\end{array}$ & \\
\hline 42.21 & 1455 & 1456 & $\alpha$-humulene & $1.92 \pm 0.13$ & $2.23 \pm 0.17$ & $1.93 \pm 0.05$ & $6.27 \pm 0.15$ & $9.42 \pm 0.48$ & $0.28 \pm 0.01$ & $2.23 \pm 0.11$ & $24.72 \pm 1.65$ & $1.39 \pm 0.06$ & $4.40 \pm 0.24$ & Std & \\
\hline 42.43 & 1457 & 1456 & (E)- $\beta$-farnesene & & & & & & & & & & & MS-RI & \\
\hline 42.53 & 1457 & 1454 & $\alpha$-patchoulene & & & & & & $0.13 \pm 0.01$ & & & & & MS-RI & \\
\hline $\begin{array}{l}43.04 \\
43.12\end{array}$ & 1458 & 1458 & $\begin{array}{l}\text { phenethyl pivalate } \\
\text { unknown } 3\end{array}$ & $2.56 \pm 0.16$ & $1.18 \pm 0.06$ & & $0.17 \pm 0.01$ & $0.55 \pm 0.04$ & $0.13 \pm 0.01$ & & & & & MS-RI & \\
\hline 42.93 & 1460 & 1460 & (Z)-methyl isoeugenol & & & & & & & & & & & MS-RI & \\
\hline 43.22 & 1460 & 1458 & alloaromadendrene & & & & $0.19 \pm 0.01$ & & $4.95 \pm 0.24$ & & & $2.60 \pm 0.16$ & $7.99 \pm 0.49$ & MS-RI & \\
\hline 43.33 & 1489 & 1490 & $\beta$-selinene & & & & & & & $3.22 \pm 0.16$ & & & & Std & \\
\hline 43.51 & 1492 & 1492 & $\gamma$-selinene & & & & & & & $3.31 \pm 0.15$ & & & & Std & \\
\hline 43.55 & 1498 & 1499 & $\alpha$-selinene & & & & $0.30 \pm 0.02$ & & $3.06 \pm 0.19$ & & & & & Std & \\
\hline 43.61 & 1500 & 1502 & bicyclogermacrene & & & & $1.45 \pm 0.06$ & & & $1.08 \pm 0.05$ & & & & Std & \\
\hline 43.68 & 1506 & 1512 & $\beta$-bisabolene & & & & & & & & & & & MS-RI & \\
\hline 44.06 & 1521 & 1520 & dihydroeugenyl butanoate & $1.39 \pm 0.63$ & $0.74 \pm 0.08$ & $1.07 \pm 0.04$ & $0.88 \pm 0.04$ & $1.62 \pm 0.07$ & $1.37 \pm 0.05$ & $0.10 \pm 0.01$ & $0.25 \pm 0.02$ & & & MS-RI & [13] \\
\hline 44.23 & 1523 & 1520 & $\delta$-cadinene & & & & & & $0.14 \pm 0.01$ & & & & & Std & \\
\hline 44.70 & 1530 & 1532 & zonarene & $1.28 \pm 0.71$ & & & $1.04 \pm 0.05$ & $0.30 \pm 0.02$ & $0.28 \pm 0.02$ & $0.43 \pm 0.03$ & $0.75 \pm 0.05$ & & & MS-RI & \\
\hline 44.87 & 1546 & 1543 & $\alpha$-calacorene & & & & & & $0.15 \pm 0.01$ & & & & & MS-RI & \\
\hline 44.92 & 1547 & 1546 & selina-3,7(11)-diene & & & & $0.31 \pm 0.02$ & & & & $0.92 \pm 0.03$ & & & MS-RI & \\
\hline 45.12 & 1548 & 1548 & (Z)-nerolidol & & & & $0.41 \pm 0.03$ & & & $0.33 \pm 0.02$ & & & & Std & \\
\hline 45.37 & 1550 & 1549 & elemol & & & & $0.28 \pm 0.02$ & & $0.09 \pm 0.01$ & & $2.29 \pm 0.12$ & & $1.84 \pm 0.13$ & MS-RI & \\
\hline 45.41 & 1551 & 1553 & ledol & & & & & & & & & & & MS-RI & \\
\hline $\begin{array}{r}45.89 \\
45.97\end{array}$ & 1578 & 1579 & 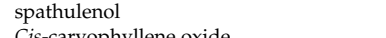 & & & & $0.16 \pm 0.01$ & & $2.38 \pm 0.13$ & $1.53 \pm 0.06$ & & & & MS-RI & \\
\hline $\begin{array}{l}45.97 \\
45.97\end{array}$ & $\begin{array}{l}1580 \\
1583\end{array}$ & 1581 & $\begin{array}{l}\text { Cis-caryophyntene oxide } \\
\text { Trans-caryophyllene oxide }\end{array}$ & $15.68+0.98$ & $383+014$ & $2.07 \pm 0.06$ & $4.37 \pm 0.14$ & $5.72+0.22$ & $10.18+0.64$ & $213 \pm 0.09$ & $1.29+0.10$ & & $0.97 \pm 0.07$ & $\begin{array}{l}\text { MS-KI } \\
\text { MS-RI }\end{array}$ & \\
\hline 46.30 & 1594 & 1595 & Cis-arteannuic alcohol & $10.00 \pm 0.90$ & & & & & & & & & $2.88 \pm 0.16$ & MS-RI & [47] \\
\hline 46.31 & 1598 & 1596 & carotol & & & & & & & & & & & MS-RI & [44] \\
\hline 46.32 & 1601 & 1600 & guaiol & & & & & & & & & & & Std & \\
\hline 46.18 & 1603 & 1603 & $\alpha$-dihydro $(10,11)$ bi sabolol & $0.35 \pm 0.02$ & & & $0.87 \pm 0.09$ & & $0.49 \pm 0.04$ & & $0.34 \pm 0.02$ & & & MS-RI & \\
\hline 46.48 & 1606 & 1608 & humulene epoxide II & $2.86 \pm 0.15$ & $0.87 \pm 0.04$ & $0.83 \pm 0.07$ & $7.26 \pm 0.68$ & $6.01 \pm 0.31$ & $4.88 \pm 0.25$ & $0.62 \pm 0.04$ & $2.54 \pm 0.11$ & & & MS-RI & [48] \\
\hline 46.80 & 1613 & 1617 & isoleptospermone & & & & & & & & & & & MS-RI & \\
\hline 46.82 & 1620 & 1623 & leptospermone & & & & & & $1.17 \pm 0.07$ & & & & & MS-RI & [49] \\
\hline 46.92 & & & unknown 4 & & & & & & & $0.36 \pm 0.02$ & & & & & \\
\hline 46.98 & 1631 & 1631 & $\begin{array}{l}\text { dihydroeugenyl pentanoate } \\
\text { unknown } 5\end{array}$ & $4.49 \pm 0.36$ & $1.06 \pm 0.06$ & $0.89 \pm 0.05$ & $2.21 \pm 0.14$ & $2.51 \pm 0.09$ & $162+010$ & & & & & MS-RI & [13] \\
\hline 47.15 & 1632 & 1634 & $\gamma$-eudesmol & & & & & & $1.02+0.10$ & & & & & Std & \\
\hline 47.23 & & & Unknwon 6 & & & & & $0.66 \pm 0.04$ & $0.58 \pm 0.03$ & & & & & & \\
\hline $\begin{array}{l}47.32 \\
47.42\end{array}$ & 1641 & 1643 & $\begin{array}{l}\text { alloaromadendrene epoxide } \\
\text { unknown } 7\end{array}$ & $1.72 \pm 0.12$ & & & $2.01 \pm 0.16$ & $1.36 \pm 0.08$ & $0.86 \pm 0.04$ & & $0.56 \pm 0.04$ & & & MS-RI & \\
\hline 47.43 & 1641 & 1641 & $5, \alpha$ caryophylla-4(14),8(15)-dien-5-ol & $1.55 \pm 0.11$ & & & $0.52 \pm 0.04$ & $0.55 \pm 0.04$ & $0.96 \pm 0.04$ & & & & & MS-RI & \\
\hline 47.55 & 1642 & 1642 & epi- $\alpha$-mu & & & & & & & & & & & MS-RI & \\
\hline 47.83 & 1644 & 1644 & $\alpha$-selinen-3,11-en-6-ol & $0.23 \pm 0.01$ & & & & & $0.27 \pm 0.02$ & $2.51 \pm 0.13$ & $0.92 \pm 0.05$ & & & MS & \\
\hline
\end{tabular}


Table 3. Cont.

\begin{tabular}{|c|c|c|c|c|c|c|c|c|c|c|c|c|c|c|c|}
\hline & & & $\begin{array}{c}\text { Genotype Number } \\
\text { Selection }\end{array}$ & $\begin{array}{c}21 \\
\text { V15 } \\
\end{array}$ & $\begin{array}{c}22 \\
\text { V16 } \\
\end{array}$ & $\begin{array}{c}23 \\
\text { V17 } \\
\end{array}$ & $\begin{array}{c}24 \\
\text { V19 } \\
\end{array}$ & $\begin{array}{c}25 \\
\text { V20 } \\
\end{array}$ & $\begin{array}{c}26 \\
\text { LAC1 } \\
\end{array}$ & $\begin{array}{c}27 \\
\text { LAC11 } \\
\end{array}$ & $\begin{array}{c}28 \\
\text { LAC31 } \\
\end{array}$ & $\begin{array}{c}29 \\
\text { BOS1 }\end{array}$ & $\begin{array}{c}30 \\
\text { BOS2 }\end{array}$ & $\mathrm{ID}^{\mathrm{z}}$ & Ref. \\
\hline Rt & $\begin{array}{c}\text { KI } \\
\text { Apolar } \\
\text { Lit. }\end{array}$ & $\begin{array}{c}\text { KI } \\
\text { Apolar }\end{array}$ & Compound & $\%$ & $\%$ & $\%$ & $\%$ & $\%$ & $\%$ & $\%$ & $\%$ & $\%$ & $\%$ & & \\
\hline 47.89 & 1660 & 1660 & $\alpha$-selinen-11-en-4 -ol & $0.64 \pm 0.05$ & & & $1.59 \pm 0.07$ & $0.31 \pm 0.02$ & $4.14 \pm 0.21$ & & & & & MS & \\
\hline 48.05 & 1663 & 1661 & epi-globulol & & & & & & $0.39 \pm 0.02$ & & & & $3.16 \pm 0.19$ & MS-RI & \\
\hline 47.97 & 1675 & 1670 & $\beta$-bisabolol & $0.78 \pm 0.05$ & & & & $0.24 \pm 0.02$ & $0.89 \pm 0.03$ & & & & & Std & \\
\hline 48.34 & 1682 & 1682 & ledene oxide II & & & & & & $0.12 \pm 0.01$ & & & & & MS-RI & {$[50]$} \\
\hline $\begin{array}{l}48.56 \\
49.22\end{array}$ & 1700 & 1713 & eudesm-7(11)-en-4-ol & & & & & & $0.06 \pm 0.01$ & & $0.29 \pm 0.02$ & & & MS-RI & [51] \\
\hline $\begin{array}{l}49.22 \\
50.59\end{array}$ & 1725 & 1738 & $\begin{array}{l}\text { unknown } 8 \\
\alpha \text {-farnesol }\end{array}$ & & & & $0.29 \pm 0.03$ & & $0.21 \pm 0.02$ & & & & & Std & \\
\hline & 1972 & 1978 & $n$-hexadecanoic acid & $0.35 \pm 0.02$ & & & & & $0.14 \pm 0.01$ & & & & & MS-RI & \\
\hline \multirow[t]{2}{*}{54.25} & 2000 & 1999 & eicosane & $0.66 \pm 0.04$ & & & $0.95 \pm 0.06$ & & $0.24 \pm 0.02$ & & & & & MS-RI & \\
\hline & & & Number of identified compounds & 32 & 23 & 20 & 45 & 31 & 52 & 24 & 30 & 16 & 15 & & \\
\hline
\end{tabular}

${ }^{\mathrm{z}}$ ID = Identification methods. MS: by comparison of the Mass spectrum with those of the computer mass libraries Adams, Nist 11 and by interpretation of the mass spectra fragmentations. RI: by comparison of retention index with those reported in literature [8,37]. Std: by comparison of the retention time and mass spectrum of available authentic standards. MS: identification of Mass spectrum. No-polar column ZB-5. Data are the mean of three replicates \pm standard deviation. ${ }^{y}$ Tentatively identified.

Table 4. Chemical composition of berry essential oil of eleven (n. 31-41) myrtle genotypes.

\begin{tabular}{|c|c|c|c|c|c|c|c|c|c|c|c|c|c|c|c|c|}
\hline & & & $\begin{array}{c}\text { Genotype Number } \\
\text { Selection }\end{array}$ & $\begin{array}{c}31 \\
\text { ORS2 } \\
\end{array}$ & $\begin{array}{l}32 \\
\text { ORS3 } \\
\end{array}$ & $\begin{array}{c}33 \\
\text { ISL3 }\end{array}$ & \begin{tabular}{|c|}
34 \\
BUD1
\end{tabular} & $\begin{array}{c}35 \\
\text { CUG11 } \\
\end{array}$ & $\begin{array}{c}36 \\
\text { ORO2 }\end{array}$ & $\begin{array}{c}37 \\
\text { ISL1 }\end{array}$ & $\begin{array}{l}38 \\
\text { SBD }\end{array}$ & $\begin{array}{l}39 \\
\text { SIN2 }\end{array}$ & $\begin{array}{c}40 \\
\text { MON5 } \\
\end{array}$ & $\begin{array}{l}41 \\
\text { PSF1 }\end{array}$ & $\mathrm{ID}^{\mathrm{z}}$ & Ref. \\
\hline Rt & $\begin{array}{c}\text { KI } \\
\text { apolar } \\
\text { Lit. }\end{array}$ & $\begin{array}{c}\text { KI } \\
\text { apolar }\end{array}$ & Compound & & & & & & & & & & & & & \\
\hline 17.88 & 939 & 937 & $\alpha$-pinene & & & & & & & & & & & $0.36 \pm 0.02$ & Std & \\
\hline 21.40 & 986 & 985 & butanoic acid, 2-methyl-2-methylpropyl ester & & & & & & & & & & & & MS-RI & [43] \\
\hline 21.6 & 1002 & 1001 & $\alpha$-phellandrene & & & & & & & & & & & & Std & \\
\hline 21.93 & 1002 & 1002 & $\delta$-2-carene & & & & & & & & & & & & Std & \\
\hline 22.25 & 1005 & 1004 & pseudolimonene & & & & & & & & & & & & MS-RI & [44] \\
\hline 22.66 & 1025 & 1021 & p-cymene & & $0.22 \pm 0.01$ & & $0.58 \pm 0.04$ & & & & $0.77 \pm 0.05$ & & & $1.03 \pm 0.06$ & Std & \\
\hline 22.90 & 1031 & 1029 & limonene & & & & $0.64 \pm 0.05$ & & & & $0.62 \pm 0.04$ & & & $0.69 \pm 0.05$ & Std & \\
\hline 23.07 & 1035 & 1031 & 1,8-cineole & & $0.57 \pm 0.04$ & $6.79 \pm 0.37$ & $2.25 \pm 0.12$ & & & & $3.12 \pm 0.22$ & $0.55 \pm 0.03$ & & $7.75 \pm 0.30$ & Std & \\
\hline 24.49 & 1060 & 1057 & $\gamma$-terpinene & & & & $0.35 \pm 0.02$ & & & & $1.41 \pm 0.11$ & & & $0.64 \pm 0.05$ & Std & \\
\hline 26.08 & 1089 & 1063 & $\alpha$-terpinolene & & & & $0.56 \pm 0.04$ & & & & $1.72 \pm 0.12$ & & & $0.92 \pm 0.06$ & Std & \\
\hline 26.55 & 1097 & 1094 & linalool & & $3.41 \pm 0.17$ & $35.10 \pm 2.36$ & $2.02 \pm 0.10$ & & & & $13.61 \pm 0.89$ & $10.02 \pm 0.51$ & $1.43 \pm 0.06$ & $2.39 \pm 0.11$ & Std & \\
\hline 26.75 & 1112 & 1108 & n-amyl isovalerate & & & & & & & & & $0.16 \pm 0.01$ & $3.10 \pm 0.16$ & $0.23 \pm 0.01$ & MS-RI & [45] \\
\hline $\begin{array}{l}28.81 \\
30.62\end{array}$ & $\begin{array}{l}1113 \\
1130\end{array}$ & $\begin{array}{l}1115 \\
1133\end{array}$ & $\begin{array}{l}\text { Trans-pinocarveol } \\
\text { cosmene }\end{array}$ & & $0.17 \pm 0.01$ & & & & & & & $0.58 \pm 0.03$ & & & MS-RI & {$[467$} \\
\hline 30.63 & 1168 & 1161 & $\begin{array}{l}\text { cosmene } \\
\text { Trans-p-matha-1(7),8-dien-2-ol }\end{array}$ & & $5.80 \pm 0.32$ & & & & & & & & & & $\begin{array}{l}\text { MSSRl } \\
\text { MS-RI }\end{array}$ & {$[46]$} \\
\hline 30.70 & 1177 & 1177 & terpinen-4-ol & & $3.79 \pm 0.13$ & & $1.30 \pm 0.06$ & & & & $3.19 \pm 0.19$ & $4.14 \pm 0.28$ & & $3.42 \pm 0.19$ & Std & \\
\hline 30.98 & 1180 & 1180 & $m$-cymen-8-ol & & & & & & & & & $0.28 \pm 0.02$ & & $0.38 \pm 0.02$ & Std & \\
\hline 31.15 & 1183 & 1181 & $p$-cymen-8-ol & & $0.95 \pm 0.06$ & & & & & & & $0.30 \pm 0.02$ & & $0.28 \pm 0.02$ & Std & \\
\hline 31.32 & 1189 & 1190 & $\alpha$-terpineol & $2.93 \pm 0.18$ & $6.90 \pm 0.34$ & $5.10 \pm 0.24$ & $3.94 \pm 0.18$ & $1.00 \pm 0.06$ & & $0.12 \pm 0.01$ & $13.93 \pm 1.03$ & $8.53 \pm 0.42$ & $2.78 \pm 0.14$ & $10.04 \pm 0.55$ & Std & \\
\hline 31.64 & 1192 & 1191 & estragole & & $0.28 \pm 0.01$ & & & & & & & $0.59 \pm 0.03$ & & $0.45 \pm 0.03$ & MS-RI & \\
\hline 32.69 & 1217 & 1213 & Trans-carveol & & & & & & & & & $0.10 \pm 0.01$ & & & MS-RI & \\
\hline 33.07 & 1230 & 1229 & nerol & & & & & & & & & $0.27 \pm 0.02$ & & & Std & \\
\hline 33.21 & 1245 & 1242 & (2-Z)-3-hexenyl isovalerate & & & & & & & & & $0.17 \pm 0.01$ & & & MS-RI & \\
\hline
\end{tabular}


Table 4. Cont.

\begin{tabular}{|c|c|c|c|c|c|c|c|c|c|c|c|c|c|c|c|c|}
\hline & & & $\begin{array}{c}\text { Genotype Number } \\
\text { Selection }\end{array}$ & $\begin{array}{c}31 \\
\text { ORS2 } \\
\end{array}$ & $\begin{array}{c}32 \\
\text { ORS3 } \\
\end{array}$ & $\begin{array}{l}33 \\
\text { ISL3 } \\
\end{array}$ & $\begin{array}{c}34 \\
\text { BUD1 } \\
\end{array}$ & $\begin{array}{c}35 \\
\text { CUG11 }\end{array}$ & $\begin{array}{c}36 \\
\text { ORO2 } \\
\end{array}$ & $\begin{array}{c}37 \\
\text { ISL1 } \\
\end{array}$ & $\begin{array}{l}38 \\
\text { SBD } \\
\end{array}$ & $\begin{array}{c}39 \\
\text { SIN2 } \\
\end{array}$ & $\begin{array}{c}40 \\
\text { MON5 }\end{array}$ & $\begin{array}{l}41 \\
\text { PSF1 }\end{array}$ & $\mathrm{ID}^{z}$ & Ref. \\
\hline Rt & $\begin{array}{c}\text { KI } \\
\text { apolar } \\
\text { Lit. }\end{array}$ & $\begin{array}{c}\mathrm{KI} \\
\text { apolar }\end{array}$ & Compound & & & & & & & & & & & & & \\
\hline 34.01 & 1246 & 1248 & carvone & & & & & & & & & & & & MS-RI & \\
\hline 34.28 & 1253 & 1255 & geraniol & $1.57 \pm 0.08$ & & & & $0.16 \pm 0.01$ & & $0.41 \pm 0.03$ & $1.29 \pm 0.87$ & $6.05 \pm 0.34$ & $1.27 \pm 0.10$ & & Std & \\
\hline 34.32 & 1257 & 1256 & linalyl acetate & & & & & & & & & & & & Std & \\
\hline 34.67 & 1267 & 1257 & geranial & & $1.80 \pm 0.07$ & & $0.98 \pm 0.05$ & & & & & & & $2.32 \pm 0.12$ & Std & \\
\hline 35.92 & 1289 & 1290 & bornyl acetate & & $0.56 \pm 0.03$ & & . & & & & & $0.13 \pm 0.01$ & & & Std & \\
\hline 36.37 & 1299 & 1312 & carvacrol & & $0.28 \pm 0.02$ & & & & & & & & & & Std & \\
\hline 34.25 & 1325 & 1322 & methyl geraniate & & & & & & & & & $0.09 \pm 0.01$ & & $0.50 \pm 0.04$ & Std & \\
\hline 37.45 & 1326 & 1326 & 6-isoprenyl-3-methoxymethoxy-3-methyl-ciclohexene (isomer) ${ }^{y}$ & & $0.19 \pm 0.01$ & & & & & & & $0.24 \pm 0.02$ & & & MS & \\
\hline 37.60 & 1326 & 1326 & 6-isoprenyl-3-methoxymethoxy-3-methyl-ciclohexene (isomer) ${ }^{y}$ & & $0.25 \pm 0.01$ & & & & & & & $0.80 \pm 0.04$ & & & MS & \\
\hline 37.70 & 1326 & 1326 & 6-isoprenyl-3-methoxymethoxy-3-methyl-ciclohexene (isomer) ${ }^{y}$ & & $0.24 \pm 0.01$ & & $0.44 \pm 0.03$ & & & & & $0.27 \pm 0.02$ & & & MS & \\
\hline 37.70 & 1332 & 1327 & myrtenyl acetate & & $0.51 \pm 0.04$ & & & & & & & $0.69 \pm 0.05$ & & & MS-RI & \\
\hline 38.14 & 1349 & 1351 & $\alpha$-terpinyl acetate & $21.10 \pm 0.96$ & $12.68 \pm 0.86$ & & $6.67 \pm 0.36$ & $3.90 \pm 0.16$ & & $1.15 \pm 0.06$ & & $17.70 \pm 1.06$ & $2.85 \pm 0.13$ & $20.25 \pm 1.03$ & MS-RI & \\
\hline $\begin{array}{l}38.41 \\
38.79\end{array}$ & $\begin{array}{l}1362 \\
1381\end{array}$ & $\begin{array}{l}1366 \\
1379\end{array}$ & $\begin{array}{l}\text { neryl acetate } \\
\text { geranyl acetate }\end{array}$ & $\begin{array}{l}0.61 \pm 0.04 \\
24.08 \pm 1.23\end{array}$ & $\begin{array}{r}0.60 \pm 0.04 \\
23.84 \pm 1.45\end{array}$ & & $\begin{array}{l}0.26 \pm 0.02 \\
10.46 \pm 0.51\end{array}$ & $0.18 \pm 0.01$ & $9.73 \pm 0.81$ & $0.57 \pm 0.07$ & & $0.91 \pm 0.06$ & & $0.40 \pm 0.02$ & 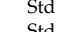 & \\
\hline 39.60 & 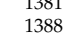 & 1388 & $\begin{array}{l}\text { geranyl acetate } \\
\beta \text {-cubebene }\end{array}$ & $24.08 \pm 1.23$ & $\begin{array}{l}23.84 \pm 1.45 \\
0.24 \pm 0.02\end{array}$ & $8.62 \pm 0.46$ & $\begin{array}{l}10.46 \pm \pm 0.51 \\
1.59 \pm 0.07\end{array}$ & $14.31 \pm 0.79$ & & $9.46 \pm 0.54$ & $10.75 \pm 0.62$ & $27.53 \pm 1.85$ & $21.42 \pm 1.05$ & $\begin{array}{l}20.09 \pm 0.96 \\
0.02 \pm 0.01\end{array}$ & $\begin{array}{l}\text { Std } \\
\text { Std }\end{array}$ & \\
\hline 39.46 & 1391 & 1395 & $\beta$-elemene & $0.63 \pm 0.05$ & $0.29 \pm 0.02$ & & $5.94 \pm 0.30$ & & & & & $1.37 \pm 0.06$ & & & Std & \\
\hline $\begin{array}{l}40.10 \\
40.25\end{array}$ & 1404 & 1401 & $\begin{array}{l}\text { methyleugenol } \\
\text { unknown 1 }\end{array}$ & $16.49 \pm 0.76$ & $9.82 \pm 0.56$ & $2.94 \pm 0.14$ & $7.03 \pm 0.41$ & $8.34 \pm 0.48$ & $0.88 \pm 0.06$ & $6.37 \pm 0.26$ & $8.53 \pm 0.46$ & $6.72 \pm 0.41$ & $7.42 \pm 0.36$ & $7.97 \pm 0.35$ & MS-RI & \\
\hline 40.69 & $\begin{array}{l}1428 \\
1435\end{array}$ & $\begin{array}{l}1430 \\
1439\end{array}$ & $\begin{array}{l}\beta \text {-caryophyllene } \\
\text { Transs } \alpha \text {-bergamotene }\end{array}$ & $8.36 \pm 0.47$ & $\begin{array}{l}0.30 \pm 0.02 \\
0.20 \pm 0.01\end{array}$ & $8.81 \pm 0.42$ & $9.91 \pm 0.58$ & $6.12 \pm 0.39$ & $1.09 \pm 0.07$ & $0.14 \pm 0.01$ & $22.26 \pm 1.85$ & $1.44 \pm 0.07$ & $35.0 \pm 2.04$ & $7.44 \pm 0.32$ & $\begin{array}{l}\text { Std } \\
\text { MS-RI }\end{array}$ & \\
\hline $\begin{array}{l}40.69 \\
41.19\end{array}$ & 1435 & $\begin{array}{l}1439 \\
1434\end{array}$ & 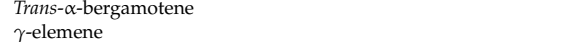 & & $0.20 \pm 0.01$ & & & $0.16 \pm 0.01$ & & & & $0.21+0.02$ & & & MS-RI & \\
\hline 41.47 & 1441 & 1443 & aromadendrene & $0.84 \pm 0.06$ & $0.61 \pm 0.04$ & & $0.41 \pm 0.03$ & $0.20 \pm 0.01$ & & & & $\begin{array}{l}0.21 \pm 0.02 \\
0.27 \pm 0.02\end{array}$ & & & $\begin{array}{l}\text { Std } \\
\text { Std }\end{array}$ & \\
\hline 41.82 & & & unknown 2 & & $0.01+0.07$ & & & & & & & & & & $\begin{array}{l}\text { Std } \\
\text { Std }\end{array}$ & \\
\hline 42.21 & 1455 & 1456 & $\alpha$-humulene & $1.64 \pm 0.05$ & $0.41 \pm 0.03$ & $26.37 \pm 1.32$ & $5.09 \pm 0.26$ & $4.14 \pm 0.28$ & & $1.35 \pm 0.06$ & $7.80 \pm 0.34$ & $0.91 \pm 0.05$ & $5.09 \pm 0.25$ & $1.86 \pm 0.09$ & Std & \\
\hline 42.43 & 1457 & 1456 & (E)- $\beta$-farnesene & & & & $0.46 \pm 0.03$ & $0.12 \pm 0.01$ & $0.99 \pm 0.06$ & & & 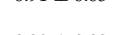 & & & MS-RI & \\
\hline 42.53 & 1457 & 1454 & $\alpha$-patchoulene & $0.53 \pm 0.04$ & $0.46 \pm 0.03$ & & $1.72 \pm 0.06$ & $0.15 \pm 0.01$ & & & & $0.39 \pm 0.03$ & & & MS-RI & \\
\hline 43.04 & 1458 & 1458 & phenethyl pivalate & $0.45 \pm 0.03$ & & & & & & & & & & & MS-RI & \\
\hline 43.12 & & & unknown 3 & & & & & & & & & & & & & \\
\hline 42.93 & 1460 & 1460 & (Z)-methyl isoeugenol & & & & & $0.15 \pm 0.01$ & & $0.10 \pm 0.01$ & & $0.13 \pm 0.01$ & & & MS-RI & \\
\hline 43.22 & 1460 & 1458 & alloaromadendrene & $3.32 \pm 0.21$ & $0.36 \pm 0.02$ & & $9.82 \pm 0.52$ & $0.75 \pm 0.05$ & & $0.98 \pm 0.06$ & & & & $0.15 \pm 0.01$ & MS-RI & \\
\hline 43.33 & 1489 & 1490 & $\beta$-selinene & & & & & & & & & $1.05 \pm 0.06$ & & & Std & \\
\hline 43.51 & 1492 & 1492 & $\gamma$-selinene & & & & & $0.28 \pm 0.02$ & & & & & & $0.31 \pm 0.02$ & Std & \\
\hline $\begin{array}{l}43.55 \\
43.61\end{array}$ & 1498 & 1499 & $\alpha$-selinene & $3.81 \pm 0.24$ & $0.37 \pm 0.02$ & & $10.04 \pm 0.53$ & $1.11 \pm 0.06$ & & $1.12 \pm 0.08$ & & & & & Std & \\
\hline $\begin{array}{l}43.61 \\
43.68\end{array}$ & $\begin{array}{l}1500 \\
1506\end{array}$ & $\begin{array}{l}1502 \\
1512\end{array}$ & $\begin{array}{l}\text { bicyclogermacrene } \\
\beta \text {-bisabolene }\end{array}$ & & & & & & $\begin{array}{l}3.97 \pm 0.19 \\
0.87+0.04\end{array}$ & $0.14 \pm 0.01$ & $1.14 \pm 0.07$ & & & & $\begin{array}{c}\text { Std } \\
\text { MS-RI }\end{array}$ & \\
\hline 44.06 & 1521 & $\begin{array}{l}1512 \\
1520\end{array}$ & $\begin{array}{l}\beta \text {-bisabolene } \\
\text { dihydroeugenyl butanoate }\end{array}$ & $1.00+0.06$ & $0.88+0.05$ & & $0.57+0.04$ & $2.17+1.15$ & $0.87 \pm 0.04$ & $0.24+0.01$ & $1.27+0.08$ & & & $0.92+0.07$ & $\begin{array}{l}\text { MS-RI } \\
\text { MS-RI }\end{array}$ & [13] \\
\hline 44.23 & 1523 & 1520 & $\delta$-cadinene & $1.00 \pm 0.00$ & $0.00 \pm 0.05$ & & $0.92 \pm 0.04$ & $1.54 \pm 0.07$ & & $0.24 \pm 0.01$ & $1.27 \pm 0.00$ & & & . & Std & 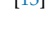 \\
\hline 44.70 & 1530 & 1532 & zonarene & & $0.73 \pm 0.04$ & & & $1.84 \pm 0.09$ & $2.60 \pm 0.15$ & & $1.46 \pm 0.09$ & & & $0.74 \pm 0.06$ & MS-RI & \\
\hline 44.87 & 1546 & 1543 & $\alpha$-calacorene & & $0.33 \pm 0.02$ & & & $0.27 \pm 0.01$ & & $0.28 \pm 0.02$ & & & & $0.71 \pm 0.05$ & MS-RI & \\
\hline 44.92 & 1547 & 1546 & selina-3,7(11)-diene & & & & $0.43 \pm 0.03$ & $1.29 \pm 0.05$ & & & $0.63 \pm 0.05$ & & & & MS-RI & \\
\hline 45.12 & 1548 & 1548 & (Z)-nerolidol & & $0.16 \pm 0.01$ & & & $1.85 \pm 0.14$ & & $0.92 \pm 0.07$ & & & & & Std & \\
\hline 45.37 & 1550 & 1549 & elemol & & & & $1.57 \pm 0.06$ & $2.11 \pm 0.13$ & $5.71 \pm 0.28$ & $0.38 \pm 0.03$ & $2.16 \pm 0.12$ & & $3.42 \pm 0.18$ & $2.30 \pm 0.12$ & MS-RI & \\
\hline 45.41 & 1551 & 1553 & ledol & & & & & & & $0.33 \pm 0.02$ & & & & & MS-RI & \\
\hline 45.89 & 1578 & 1579 & spathulenol & $1.74 \pm 0.07$ & $3.40 \pm 0.19$ & & $0.61 \pm 0.05$ & $1.21 \pm 0.06$ & $2.86 \pm 0.16$ & $7.84 \pm 0.36$ & & $0.90 \pm 0.05$ & & $0.29 \pm 0.02$ & MS-RI & \\
\hline $\begin{array}{l}45.97 \\
45.97\end{array}$ & $\begin{array}{l}1580 \\
1583\end{array}$ & $\begin{array}{l}1581 \\
1583\end{array}$ & $\begin{array}{l}\text { Cis-caryophyllene oxide } \\
\text { Trans-caryophyllene oxide }\end{array}$ & $5.84 \pm 0.26$ & $2.36 \pm 0.15$ & & $2.55 \pm 0.16$ & $\begin{array}{l}0.95 \pm 0.05 \\
9.50 \pm 0.41\end{array}$ & $25.69 \pm 1.03$ & $9.20 \pm 0.58$ & $2.91 \pm 0.16$ & $1.11 \pm 0.05$ & & $1.83 \pm 0.10$ & $\begin{array}{l}\text { MS-RI } \\
\text { MS-RI }\end{array}$ & \\
\hline 46.30 & 1594 & 1595 & Cis-arteannuic alcohol & & $0.24 \pm 0.01$ & & & & & & & & & & MS-RI & [47] \\
\hline
\end{tabular}


Table 4. Cont

\begin{tabular}{|c|c|c|c|c|c|c|c|c|c|c|c|c|c|c|c|c|}
\hline & & & $\begin{array}{c}\text { Genotype Number } \\
\text { Selection }\end{array}$ & $\begin{array}{c}31 \\
\text { ORS2 } \\
\end{array}$ & $\begin{array}{c}32 \\
\text { ORS3 } \\
\end{array}$ & $\begin{array}{l}33 \\
\text { ISL3 } \\
\end{array}$ & $\begin{array}{c}34 \\
\text { BUD1 } \\
\end{array}$ & $\begin{array}{c}35 \\
\text { CUG11 } \\
\end{array}$ & $\begin{array}{c}36 \\
\text { ORO2 } \\
\end{array}$ & $\begin{array}{l}37 \\
\text { ISL1 } \\
\end{array}$ & $\begin{array}{l}38 \\
\text { SBD } \\
\end{array}$ & $\begin{array}{c}39 \\
\text { SIN2 } \\
\end{array}$ & $\begin{array}{c}40 \\
\text { MON5 }\end{array}$ & $\begin{array}{l}41 \\
\text { PSF1 } \\
\end{array}$ & $\mathrm{ID}^{\mathrm{z}}$ & Ref. \\
\hline Rt & $\begin{array}{c}\mathrm{KI} \\
\text { apolar } \\
\text { Lit. }\end{array}$ & $\begin{array}{c}\text { KI } \\
\text { apolar }\end{array}$ & Compound & & & & & & & & & & & & & \\
\hline 46.31 & 1598 & 1596 & carotol & & $0.22 \pm 0.01$ & & & $0.62 \pm 0.05$ & & & & & & & MS-RI & {$[44]$} \\
\hline 46.32 & 1601 & 1600 & $\begin{array}{l}\text { guaiol } \\
\alpha \text {-dibhdro } 10.11 \mathrm{bi} \text { sabolol }\end{array}$ & & $0.46 \pm 0.03$ & & & $0.28 \pm 0.02$ & & $2.03 \pm 0.10$ & & $0.15 \pm 0.01$ & $9.91 \pm 0.55$ & & Std & \\
\hline 46.18 & 1603 & 1603 & $\alpha$-dihydro $(10,11)$ bi sabolol & & & & & $0.74 \pm 0.06$ & & $2.14 \pm 0.15$ & & & & & MS-RI & \\
\hline 46.48 & 1606 & 1608 & humulene epoxide II & $0.55 \pm 0.04$ & $0.97 \pm 0.05$ & $5.54 \pm 0.22$ & $0.81 \pm 0.06$ & $4.68 \pm 0.21$ & $4.94 \pm 0.08$ & $15.43 \pm 0.96$ & $0.62 \pm 0.05$ & $0.43 \pm 0.03$ & & $0.36 \pm 0.02$ & MS-RI & [48] \\
\hline 46.80 & 1613 & 1617 & isoleptospermone & & & & & & & & & & & & MS-RI & \\
\hline 46.82 & 1620 & 1623 & $\begin{array}{l}\text { leptospermone } \\
\text { unknown } 4\end{array}$ & & $0.26 \pm 0.01$ & & & & & & & & $199+012$ & & MS-RI & [49] \\
\hline $\begin{array}{l}46.92 \\
46.98 \\
4706\end{array}$ & 1631 & 1631 & dihydroeugenyl pentanoate & & & & $0.80 \pm 0.05$ & $3.48 \pm 0.17$ & $1.05 \pm 0.04$ & $0.55 \pm 0.04$ & & & $1.99+0.12$ & $0.23 \pm 0.01$ & MS-RI & [13] \\
\hline $\begin{array}{l}47.06 \\
47.15 \\
4723\end{array}$ & 1632 & 1634 & $\begin{array}{l}\text { unknown } 5 \\
\gamma \text {-eudesmol } \\
\text { Unknwon } 6\end{array}$ & & $0.47 \pm 0.03$ & & & $2.21 \pm 0.13$ & & & & & & & Std & \\
\hline $\begin{array}{l}47.32 \\
47.42\end{array}$ & 1641 & 1643 & $\begin{array}{l}\text { alloaromadendrene epoxide } \\
\text { unknown } 7\end{array}$ & $0.61 \pm 0.05$ & $0.35 \pm 0.02$ & & & $1.68 \pm 0.07$ & $1.47 \pm 0.06$ & $4.44 \pm 0.21$ & & $0.05 \pm 0.01$ & & & MS-RI & \\
\hline 47.43 & 1641 & 1641 & $5, \alpha$ caryophylla-4(14),8(15)-dien-5-ol & & & & $0.52 \pm 0.04$ & $2.19 \pm 0.12$ & $2.03 \pm 0.13$ & $2.41 \pm 0.10$ & & $0.15 \pm 0.01$ & & $0.33 \pm 0.02$ & MS-RI & \\
\hline 47.55 & 1642 & 1642 & epi- $\alpha$-muurolol & & & & & $0.83 \pm 0.07$ & & & & & & & MS-RI & \\
\hline 47.83 & 1644 & 1644 & $\alpha$-selinen-3,11-en-6-ol & $0.31 \pm 0.02$ & $0.77 \pm 0.03$ & & $0.65 \pm 0.05$ & $3.06 \pm 0.19$ & $6.80 \pm 0.37$ & $1.80 \pm 0.09$ & & & & $0.59 \pm 0.04$ & MS & \\
\hline 47.89 & 1660 & 1660 & $\alpha$-selinen-11-en-4 -ol & $1.18 \pm 0.06$ & $0.20 \pm 0.01$ & & $5.97 \pm 0.29$ & $1.53 \pm 0.07$ & $12.26 \pm 0.62$ & $8.36 \pm 0.27$ & & $0.81 \pm 0.04$ & & $0.13 \pm 0.01$ & MS & \\
\hline 48.05 & 1663 & 1661 & epi-globulol & & & & & & & $0.30 \pm 0.02$ & & $0.03 \pm 0.01$ & & & MS-RI & \\
\hline 47.97 & 1675 & 1670 & $\begin{array}{l}\beta \text {-bisabolol } \\
\text { ledene oxide II }\end{array}$ & & & & $0.42 \pm 0.03$ & $1.35 \pm 0.06$ & $0.95 \pm 0.04$ & $0.89 \pm 0.04$ & & & $1.16 \pm 0.07$ & & $\begin{array}{c}\text { Std } \\
\text { MS-RI }\end{array}$ & \\
\hline 48.34 & 1682 & 1682 & ledene oxide II & & $0.53 \pm 0.03$ & & & $0.33 \pm 0.02$ & $0.94 \pm 0.05$ & $0.65 \pm 0.05$ & & & & $0.13 \pm 0.01$ & MS-RI & {$[50]$} \\
\hline 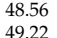 & 1700 & 1713 & $\begin{array}{l}\text { eudesm-7(11)-en-4-ol } \\
\text { unknown } 8\end{array}$ & & $0.24 \pm 0.01$ & & & $0.62 \pm 0.05$ & $1.41 \pm 0.06$ & $0.22 \pm 0.01$ & $0.36 \pm 0.02$ & & & & MS-RI & [51] \\
\hline 50.59 & 1725 & 1738 & $\begin{array}{l}\text { unknown } 8 \\
\alpha \text {-farnesol }\end{array}$ & & $1.30 \pm 0.06$ & & & $0.59 \pm 0.04$ & $1.59 \pm 0.06$ & $0.33 \pm 0.02$ & & & & & Std & \\
\hline 51.29 & 1972 & 1978 & $n$-hexadecanoic acid & & & & & $0.23 \pm 0.01$ & & $0.07 \pm 0.01$ & & & & & MS-RI & \\
\hline \multirow[t]{2}{*}{54.25} & 2000 & 1999 & eicosane & & & & & $0.19 \pm 0.01$ & & $0.85 \pm 0.04$ & & & & & MS-RI & \\
\hline & & & Number of identified compounds & 21 & 47 & 8 & 35 & 43 & 20 & 34 & 21 & 39 & 13 & 36 & & \\
\hline
\end{tabular}

${ }^{\mathbf{z}}$ ID = Identification methods. MS: by comparison of the Mass spectrum with those of the computer mass libraries Adams, Nist 11 and by interpretation of the mass spectra fragmentations. RI: by comparison of retention index with those reported in literature [8,37]. Std: by comparison of the retention time and mass spectrum of available authentic standards. MS: identification of Mass spectrum. No-polar column ZB-5. Data are the mean of three replicates \pm standard deviation. ${ }^{\mathrm{y}}$ Tentatively identified. 
Finally, we detected both the presence of previously described chemotypes or associations of main components and the original nature of some chemical profiles not previously reported for myrtle berries. This was the case of RUM12 with prevalence of metyleugenol, trans-caryophyllene oxide and dihydroeugenyl pentanoate, a recently described compound for the myrtle leaf and flower essential oils [13], and 38 other compounds in a very complex essential oil. Other genotypes showed chemical profiles of the essential oils absolutely originals: CPT3 with neryl acetate, $\alpha$-humulene and $\alpha$-selinene as main components; V7 with $\alpha$-humulene, Trans-caryophyllene oxide and humulene epoxide II; and ORO2 with neryl acetate, Trans-caryophyllene oxide, and selinene-11-en-4- $\alpha$-ol.

Application of multivariate analysis showed that the main components of the variance separate fairly genotypes in two groups based on essential oil chemical composition (Figure 2). We easily distinguish a chemotype that spreads in the South-East of the Sardinia and in all the localities above $300 \mathrm{~m}$ of altitude (white symbols) and another spreading all over the other localities where the genotypes were selected [41].

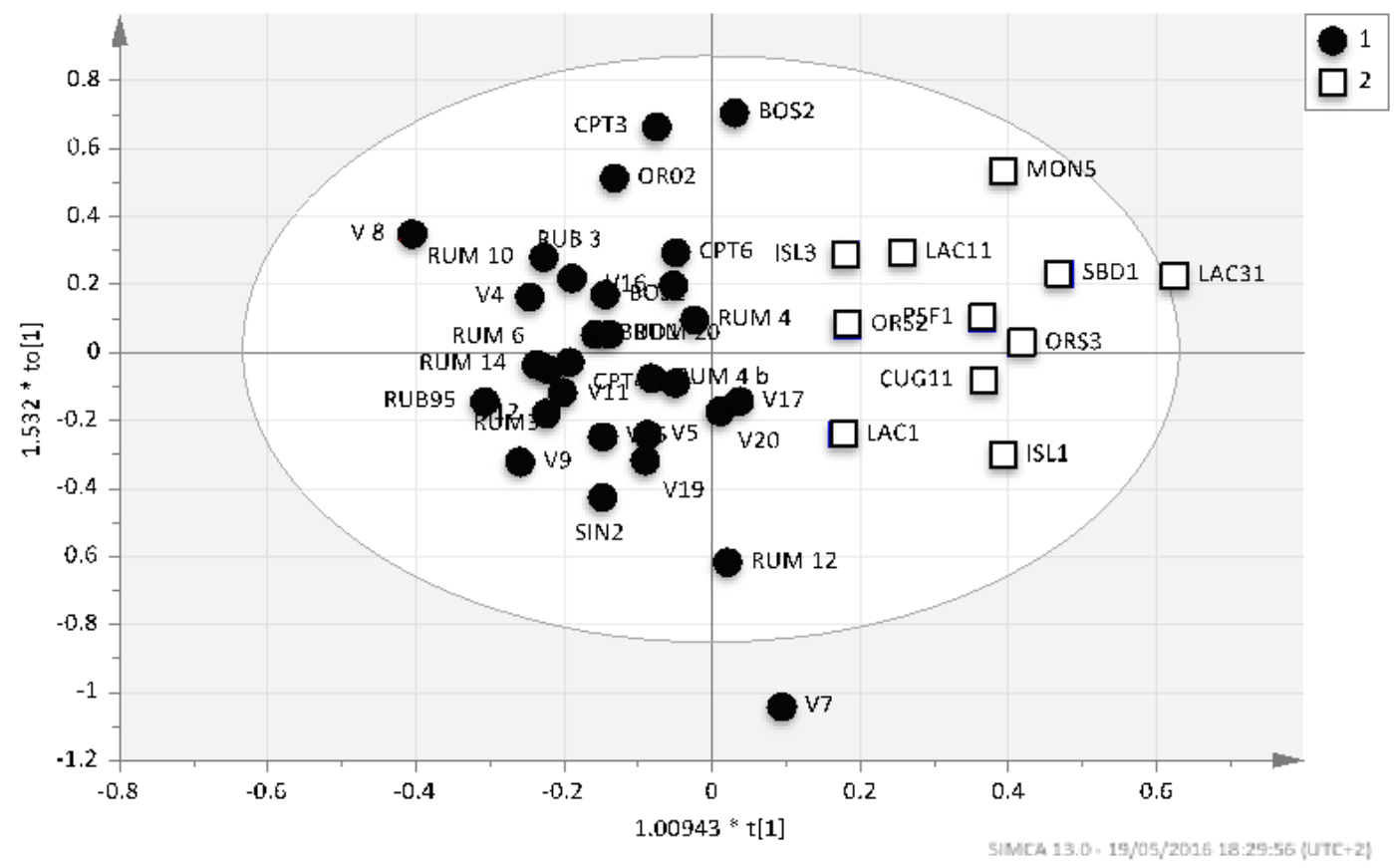

Figure 2. Genotypes distribution according to the two main components of variance as obtained by statistical multivariate analysis.

\section{Experimental Section}

\subsection{Plant Materials and Essential Oils Distillation}

The fruits have been harvested in the educational and experimental farm "Antonio Milella" located in San Quirico (Fenosu-Oristano, Central Wester Sardinia, Italy) in December 2015 when fully ripe. Among the 47 cultivars, only 5 are belonging to the variety leucocarpa DC, that means with white-yellow or withe-green fruits, the other 42 are belonging to the variety melanocarpa DC, that means with black-blue or purple fruits. The considered selections originate from different localities of Sardinia [5]. At least 15 plants represented every candidate clone. Mulas M. identified the analyzed plants. Voucher specimens have been deposited at the Herbarium SASSA (Sassari) of the Department of Chemistry and Pharmacy, University of Sassari under a collective number 514.

To avoid a harvesting not representative we collected the fruits all at the same phenological stage (fully ripe) making sure to take plant material around all plants collecting material from the top, from 
the sides and from the base of threes. In the laboratory, the plant material was cleaned from other foreign parts (little branches, lives) and the samples were made as uniform as possible.

From every cultivar where collected about $2 \mathrm{~kg}$ of fruits and divided into three parts to replicate the analyses. After harvest, the clean fruits were kept in refrigerator at $-20^{\circ} \mathrm{C}$ until their extraction. Every sample of berries was chopped using a blender at low speed and the essential oil samples were obtained from the chopped berries by hydrodistillation for $4 \mathrm{~h}$ using a Clevenger-type apparatus. For every selection three extractions were performed. The extraction yields calculated as $\mathrm{g} \cdot \mathrm{kg}^{-1}$ of fresh material are reported in Figure 1 . The oils were stored in sealed vials, at $-20^{\circ} \mathrm{C}$, ready for the chemical analysis.

\subsection{Gas Chromatography-Mass Spectrometry (GC/MS) Analysis}

GC: Three replicates of each sample were analyzed by using a Hewlett-Packard Model 5890A GC, equipped with a flame ionization detector and fitted with a $60 \mathrm{~m} \times 0.25 \mathrm{~mm}$ (I.D.), thickness $0.25 \mu \mathrm{m}$ ZB-5 fused silica capillary column (Phenomenex, Torrance CA, USA). Injection port and detector temperatures were maintained at $280^{\circ} \mathrm{C}$.

The column temperature was programmed from $50{ }^{\circ} \mathrm{C}$ to $135^{\circ} \mathrm{C}$ at $5{ }^{\circ} \mathrm{C} / \mathrm{min}(1 \mathrm{~min}), 5^{\circ} \mathrm{C} / \mathrm{min}$ up $225^{\circ} \mathrm{C}(5 \mathrm{~min}), 5^{\circ} \mathrm{C} / \mathrm{min}$ up $260^{\circ} \mathrm{C}$ and then held for $10 \mathrm{~min}$.

Samples of $0.2 \mu \mathrm{L}$ (volume injection) were analyzed, diluted in hexane using 2,6-dimethylphenol as internal standard. Injection was performed using a split/splitless automatic injector HP 7673 and helium as carrier gas. Several measurements of peak areas were performed with a HP workstation with a threshold set to 0 and peak width to 0.02 . The quantization of each compound was expressed as absolute weight percentage using internal standard and response factors (RFs). The detector RFs were determined for key components relative to 2,6-dimethylphenol and assigned to other components based on functional group and/or structural similarity, since oxygenated compounds have lower detectability by FID (Flame Ionization Detector) than hydrocarbons. The standards (Sigma-Aldrich, Fluka and Merck grade) were $>95 \%$ also, and actual purity was checked by GC. Several response factor solutions were prepared that consisted of only four or five components (plus 2,6-dimethylphenol) to prevent interference from trace impurities. It is known that the oxygenated compounds have a lower sensitivity than the hydrocarbons to FID, we have calculated the response factor using a standard mixture of $\alpha$-pinene, $\alpha$-terpineol, neral, geranial, geranyl acetate and caryophyllene; in this mixture terpene accounted for $92 \%$ of the mixture, aldehydes $5 \%$ and alcohols, esters and sesquiterpenes $1 \%$ each. In our analyses we obtained that the RF of hydrocarbons was equal to 1 while for alcohols it was 0.80 and for esters 0.71 . For this reason, we have multiplied the experimental data obtained for the following correction factors: hydrocarbons for 1, aldehydes and ketones for 1.24, alcohols for 1.28 and esters for 1.408 .

GC/MS: MS analyses were carried out with an Agilent Technologies model 7820A connected with a MS detector 5977E MSD (Agilent), and using the same conditions and column described above. The column was connected to the ion source of the mass spectrometer. Mass units were monitored from 10 to $900 \mathrm{AMU}$ at $70 \mathrm{eV}$. In the identification procedure we considered only the peaks from 40 to 900 AMU.

The identification of constituents was based on comparison of the $R_{\mathrm{t}}$ values and mass spectra with those obtained from authentic samples and/or the Nist and Wiley library spectra, or on the interpretation of the EI-fragmentation of the molecules [52,53].

\subsection{Statistical Analysis}

Oil yield data were processed for ANOVA by means of the software MSTAT-C and mean separation of was performed by application of the Tukey's test at $p \leq 0.05$ level of significance.

Data were submitted to multivariate statistical evaluation. Prior to chemometric analysis, setting the total integral areas to 100 normalized the data and the generated ASCII file was imported into 
Microsoft EXCEL for the addition of labels. The matrix was imported into SIMCA-P software version 12.0, (Umetrics AB, Umeå, Sweden) for statistical analysis.

\section{Conclusions}

The essential oil content of myrtle berries is quite low with respect to the yields that may be recovered by leaves or flowers of the same plant. Moreover, in six genotypes yields obtained with the hydrodistillation extraction system were insufficient for sample analysis. However, the importance of the essential oil composition for organoleptic properties of myrtle berries or of food and medicinal products obtained from their biomass is fundamental.

Among the main constituents of the myrtle essential oils geranyl acetate is a compound with a floral or fruity rose aroma. Geranyl acetate is soluble in alcohol and is used as a flavoring ingredient where a sweet fruity or citrus aroma is desired. Many uses are also reported for 1,8-cineole as fragrance and flavoring agent in foods, candies, cough drops, and personal care products [54,55]. This compound is the chief constituent of the oil of eucalyptus and was also found in essential oils of laurel, rosemary, and many other plants. $\alpha$-terpinyl acetate and $\alpha$-terpineol have pleasant odor similar to lilac and are common ingredients in perfumes, cosmetics, and flavors [56]. Linalool is a fragrant monoterpene alcohol found in the essential oils of numerous aromatic plants. Linalool is largely used as fragrance component in perfumes, cosmetics, soaps, and detergents but also as flavoring agent in foods. Methyl eugenol is mainly used as fragrance ingredient in perfumes, toiletries, detergents, and flavor ingredient in baked goods. This substance is reasonably anticipated to be a human carcinogen [57]. $\beta$-caryophyllene and Trans-caryophyllene are natural bicyclic sesquiterpenes that are constituents of many essential oils. $\beta$-caryophyllene and Trans-caryophyllene are two of the chemical compounds that contributes to the spiciness of black pepper [58]. $\alpha$-humulene and humulene epoxide II are components of the essential oil from the flowering cone of the hops plant (Humulus lupulus), from which derives they names.

Most value of the myrtle products is on their fragrance and permanence of the aromatic compounds of the berries in the processed foods, such as the typical myrtle liqueurs $[5,11]$. The research carried out provides new information on the recurrence of some aromatic profiles in the genotypes selected from the wild populations growing in Sardinia, and the directions for the possible replication in the cultivation of the candidate clones showing the most appreciable chemical composition of the berry essential oil. Considering the increasing development of the myrtle as a new crop, in the next future will be possible to increase the quality value of yielded biomasses also by combining the aromatic profiles of the cultivated clones, to obtain the most appreciated or beneficial results.

Author Contributions: M.U. and M.M. (Maurizio Mulas) conceived and designed the research; M.M. (Maurizio Mulas) provided the sample collection; M.U., M.M. (Mauro Marchetti) and N.C. performed the sample extraction, analysis and data processing; M.U., M.M. (Mauro Marchetti) and M.M. (Maurizio Mulas) wrote the paper.

Funding: The research has been supported by the Regione Autonoma della Sardegna (Special Grant L.R. 7/2007-2012: "The myrtle: genetics and metabolomics, an integrated approach for industry development").

Conflicts of Interest: The authors declare no conflict of interest.

\section{References}

1. Sumbul, S.; Ahmed, M.A.; Asif, M.; Akhtar, M. Myrtus communis Linn. A review. Indian J. Nat. Prod. Resour. 2011, 2, 395-402.

2. Available online: http://www.tropicos.org/Name/22102175 (accessed on 23 January 2015).

3. Nassar, M.I.; Aboutabl, E.A.; Ahmad, R.F.; El-Khrisy, E.-D.A.; Ibrahim, K.M.; Sleem, A.A. Secondary metabolites and bioactivities of Myrtus communis. Pharmacognosy Res. 2010, 2, 325-329. [CrossRef] [PubMed]

4. The Plant List. 2013. Version 1.1. Published on the Internet. Available online: http:/ /www.theplantlist.org/ (accessed on 1 January 2013).

5. Mulas, M. The myrtle (Myrtus communis L.) case, from a wild shrub to a new fruit crop. Acta Hortic. 2012, 948, 235-242. [CrossRef] 
6. Lawrence, B.M.; Terhune, S.J.; Hogg, J.W. Essential oil and their consituents. V. Oil of Myrtus communis. Amer. Perfum. 1970, 85, 53-55.

7. Lawrence, B.M. Progress in essential oils. Perfum. Flav. 1996, 21, 57-67.

8. Flamini, G.; Cioni, P.L.; Morelli, I.; Maccioni, S.; Baldini, R. Phytochemical typologies in some populations of Myrtus communis L. on Capriome promontory (East Liguria, Italy). Food Chem. 2004, 85, 599-604. [CrossRef]

9. Vidrich, V.; Franci, M.; Michelozzi, M.; Fusi, P. Variabilità della composizione di olii essenziali in diverse provenienze italiane di Myrtus communis L. Ital. J. For. Mt. Environ. 2006, 61, 87-92.

10. Pirisino, G.; Mulè, A.; Moretti, M.D.L.; Satta, M. Studio della resa e della composizione chimica dell'olio essenziale di Myrtus communis L. spontaneo di Cuglieri (Sardegna). Rivista italiana EPPOS 1996, 19, 159-169.

11. Tuberoso, C.I.G.; Barra, A.; Angioni, A.; Sarritzu, E.; Pirisi, F.M. Chemical composition of volatiles in Sardinian myrtle (Myrtus communis L.) alcoholic extracts and essential oils. J. Agric. Food Chem. 2006, 54, 1420-1426. [CrossRef] [PubMed]

12. Mulas, M.; Melis, R.A.M. Essential oil compositiom of myrtle (Myrtus communis L.) leaves. J. Herbs Spices Med. Plants. 2011, 17, 21-34. [CrossRef]

13. Usai, M.; Marchetti, M.; Mulas, M. Chemical composition of essential oils of leaves and flowers from five cultivars of myrtle (Myrtus communis L.). J. Essent. Oil Res. 2015, 27, 465-476. [CrossRef]

14. Bradesi, P.; Tomi, F.; Casanova, J.; Costa, J.; Bernardini, A.F. Chemical composition of myrtle leaf essential oil from Corsica (France). J. Essent. Oil Res. 1997, 9, 283-288. [CrossRef]

15. Jamoussi, B.; Romdhane, M.; Abderraba, A.; Ben Hassine, B.; El Gadri, A. Effect of harvest time on the yield and composition of Tunisian myrtle oils. Flavour Fragr. J. 2005, 20, 274-277. [CrossRef]

16. Messaoud, C.; Zaouali, Y.; Salah, A.B.; Khoudja, M.L.; Boussaid, M. Myrtus communis in Tunisia: variability of the essential oil composition in natural populations. Flavour Fragr. J. 2005, 20, 577-582. [CrossRef]

17. Wannes, W.A.; Mhamdi, B.; Marzouk, B. GC comparative analysis of leaf essential oils from two myrtle varieties at different phenological stages. Chromatographia 2009, 69, 145-150. [CrossRef]

18. Moghrani, H.; Maachi, R. Valorization of Myrtus communis essential oil by steam driving distillation. Asian J. Sci. Res. 2008, 1, 518-524. [CrossRef]

19. Bouzabata, A.; Castola, V.; Bighelli, A.; Abed, L.; Casanova, J.; Tomi, F. Chemical variability of Algerian Myrtus communis L. Chem. Biodivers. 2013, 10, 129-137. [CrossRef] [PubMed]

20. Gardeli, C.; Papageorgiou, V.; Mallouchos, A.; Theodosis, K.; Komaitis, M. Essential oil composition of Pistacia lentiscus L. and Myrtus communis L.: Evaluation of antioxidant capacity of methanolic extracts. Food Chem. 2008, 107, 1120-1130. [CrossRef]

21. Akin, M.; Aktumsek, A.; Nostro, A. Antibacterial activity and composition of the essential oils of Eucalyptus camaldulensis Dehn. and Myrtus communis L. growing in Northern Cyprus. Afr. J. Biotechnol. 2010, 9, 531-535.

22. Mimica-Dukić, N.; Bugarin, D.; Grbović, S.; Mitić-Ćulafić, D.; Vuković-Gačić, B.; Orčić, D.; Jovin, E.; Couladis, M. Essential oil of Myrtus communis as a potential antioxidant and antimutagenic agents. Molecules 2010, 15, 2759-2770. [CrossRef] [PubMed]

23. Jerkovic, I.; Radonic, A.; Borcic, I. Comparative study of leaf, fruit and flower essential oils of Croatian Myrtus communis (L.) during a one-year vegetative cycle. J. Essent. Oil Res. 2002, 14, 266-270. [CrossRef]

24. Yadegarina, D.; Gachkar, L.; Rezaei, M.B.; Taghizadeh, M.; Astaneh, S.A.; Rasooli, I. Biochemical activities of Iranian Mentha piperita L. and Myrtus communis L. essential oils. Phytochemistry 2006, 67, 1249-1255. [CrossRef] [PubMed]

25. Weyerstahl, P.; Marschall, H.; Rustaiyan, A. Constituents of the essential oil of Myrtus communis L. from Iran. Flavour Fragr. J. 1994, 9, 333-337. [CrossRef]

26. Hashemi, P.; Abolghasemi, M.M.; Ahmadi, S.; Ghiasvant, A.R. Headspace-solvent microextraction for identification of volatile components of Myrtus communis L. Acta Chromatogr. 2009, 21, 139-149. [CrossRef]

27. Moradi, M.; Kayhaii, M.; Ghiasvand, A.R.; Shadabi, S.; Salehinia, A. Composition of headspace solid-phase microextraction and hydrodistillationfor chemical screening of volatiles in Myrtus communis L. Phytochem. Anal. 2012, 23, 379-386. [CrossRef] [PubMed]

28. Rahimmalek, M.; Mirzakhani, M.; Pirbalouti, A.G. Essential oil variation among 21 wild myrtle (Myrtus communis L.) populations collected from different geographical regions in Iran. Ind. Crops Prod. 2013, 51, 328-333. [CrossRef]

29. Ghasemi Pirbalouti, A.; Craker, L.E.J. Diversity in chemical compositions of essential oil of myrtle leaves from various natural habitats in south and southwest Iran. For. Res. 2015, 26, 971-981. [CrossRef] 
30. Zomorodian, K.; Morein, M.; Lori, Z.G.; Ghasemi, Y.; Rahimi, J.M.; Bandegani, A.; Pakshir, K.; Bazargani, A.; Mirzamohammadi, S.; Abbasi, N. Chemical composition and antimicrobical activities of the essential oil from Myrtus communis leaves. J. Essent. Oil Bear. Pl. 2013, 16, 76-84. [CrossRef]

31. Alipour, G.; Dashti, S.; Hosseinzadeh, H. Review of pharmacological effects of Myrtus communis L. and its active constituents. Phytother. Res. 2014, 28, 1125-1136. [CrossRef] [PubMed]

32. Aleksic, V.; Knezevic, P. Antimicrobial and antioxidative activity of extracts and essential oils of Myrtus communis L. Microbiol. Res. 2014, 169, 240-254. [CrossRef] [PubMed]

33. Fenu, G.; Foddai, M.; Carai, A.; Pirino, A.; Usai, M. Therapeuthic properties of myrtle oil: An in vitro study on human nasal mucosa cells. Int. J. Essen. Oil Ther. 2008, 2, 21-25.

34. Boelens, M.H.; Jimenez, R. The chemical composition of Spanish myrtle oil. Part II. J. Essent. Oil Res. 1992, 4, 349-353. [CrossRef]

35. Mazza, G. Gas chromatographic-mass spectrometric investigation of the volatile components of myrtle berries (Myrtus communis L.). J. Chromatogr. 1983, 264, 304-311. [CrossRef]

36. Pereira, P.C.; Cebola, M.J.; Bernardo-Gil, M.G. Evolution of the yields and composition of essential oil from Portuguese Myrtle (Myrtus communis L.) through the vegetative cycle. Molecules 2009, 14, 3094-3105. [CrossRef] [PubMed]

37. Messaoud, C.; Boussaid, M. Myrtus communis berry color morphs: a comparative analysis of essential oils, fatty acids, phenolic compounds, and antioxidant activities. Chem. Biodiver. 2011, 8, 300-310. [CrossRef] [PubMed]

38. Brada, M.; Tabti, N.; Boutoumi, H.; Wathelet, J.P.; Lognay, G. Composition of the essential oil of leaves and berries of Algerian myrtle (Myrtus communis L.). J. Essent. Oil Res. 2012, 24, 1-3. [CrossRef]

39. Kafkas, E.; Güney, M.; Sadighazadi, S.; Yıldırım, H.; Kefayati, S. Volatile compounds of selected white and black myrtle (Myrtus communis L.) types from Mediterranean region of Turkey. J. Med. Plants Res. 2013, 7, 1244-1248.

40. Melito, S.; Chessa, I.; Erre, P.; Podani, J.; Mulas, M. The genetic diversity of Sardinian myrtle (Myrtus communis L.) populations. Electron. J. Biotechnol. 2013, 16, 6. [CrossRef]

41. Melito, S.; Fadda, A.; Rapposelli, E.; Mulas, M. Genetic diversity and population structure of Sardinian myrtle (Myrtus communis L.) selections as obtained by AFLP markers. HortScience 2014, 49, 531-537.

42. Melito, S.; Dessena, L.; Sale, L.; Mulas, M. Genetic diversity and population structure of wild Sardinian myrtle (Myrtus communis L.) genotypes from different microclimatic areas. Aust. J. Crop Sci. 2017, 11, 1488-1496. [CrossRef]

43. Mierendorff, H.G.; Stahl-Biskup, E.; Posthumus, M.A.; van Beek, T.A. Composition of commercial Cape chamomile oil (Eriocephalus punctulatus). Flavour Fragr. J. 2003, 18, 510-514. [CrossRef]

44. Flamini, G.; Cioni, P.L.; Morelli, I.; Bader, A. Essential oils of the aerial parts of three Salvia species from Jordan: Salvia lanigera, S. spinosa and S. syriaca. Food Chem. 2007, 100, 732-735. [CrossRef]

45. Liu, F.; Liang, Y.; Cao, C.; Zhou, N. QSPR study of GC retention indices for saturated esters on seven stationary phases based on novel topological indices. Talanta 2007, 72, 1307-1315. [CrossRef] [PubMed]

46. Ali, N.A.A.; Wurster, M.; Arnold, N.; Teichert, A.; Schmidt, J.; Lindequist, U.; Wessjohann, L. Chemical composition and biological activities of essential oils from the oleogum resins of three endemic soqotraen Boswellia species. Rec. Nat. Prod. 2008, 2, 6-12.

47. Mosayebi, M.; Amin, G.; Arzani, H.; Azarnivand, H.; Maleki, M.; Shafaghat, A. Effect of habitat on essential oil of Achillea filipendula L. in Iran. Asian J. Plant Sci. 2008, 7, 779-781. [CrossRef]

48. Quijano, C.E.; Salamanca, G.; Pino, J.A. Aroma volatile constituents of Colombian varieties of mango (Mangifera indica L.). Flavour Fragr. J. 2007, 22, 401-406. [CrossRef]

49. Batista-Pereira, L.G.; Fernandes, J.B.; Correa, A.G.; da Silva, M.F.G.F.; Vieira, P.C. Electrophysiological responses of eucalyptus brown looper Thyrinteina arnobia to essential oils of seven Eucalyptus species. J. Braz. Chem. Soc. 2006, 17, 555-561. [CrossRef]

50. Fakhari, A.R.; Sonboli, A.; Heydari, R. Composition of the essential oil of Rhabdosciadium strausii from Iran. Chem. Nat. Compd. 2005, 41, 413-414. [CrossRef]

51. Ramírez, J.; Gilardoni, G.; Ramón, E.; Tosi, S.; Picco, A.M.; Bicchi, C.; Vidari, G. Phytochemical study of the equadorian species Lepechinia mutica (Benth.). Epling and high antifungal activity of carnosol against Pyricularia oryzae. Pharmaceuticals 2018, 11, 33. [CrossRef] [PubMed]

52. NIST2011. Library of Mass Spectra; Agilent Technologies Co.: Palo Alto, CA, USA, 2011. 
53. Adams, R.P. Identification of Essential Oil Components by Gas Chromatography/Quadrupole Mass Spectrometry, 3rd ed.; Allured Publ. Corp.: Carol Stream, IL, USA, 2001.

54. Opdyke, D.L.J. Monographs of fragrance raw materials: Eucalyptol. Food Cosmet. Toxicol. 1975, 13, $105-106$. [CrossRef]

55. De Vincenzi, M.; Silano, M.; De Vincenzi, A.; Maialetti, F.; Scazzocchio, B. Costituents of aromatic plants: eucalyptol. Fitoterapia 2002, 73, 269-275. [CrossRef]

56. Wagner, A. Manufacture of terpineol. Manuf. Chem. Aerosol News 1951, 22, 153-155. [PubMed]

57. Smith, R.L.; Adams, T.B.; Doull, J.; Feron, V.J.; Goodman, J.I.; Marnett, L.J.; Portoghese, P.S.; Waddel, W.J.; Wagner, B.M.; Rogers, A.E.; et al. Safety assessment of allyalkoxybenzene derivatives used as flavouring substances -methyl eugenol and estragole. Food Chem. Toxicol. 2002, 40, 851-870. [CrossRef]

58. Jirovetz, L.; Buchbauer, G.; Ngassoum, M.B.; Geissler, M. Aroma compound analysis of Piper nigrum and Piper guineense essential oils from Cameroon using solid-phase microextraction-gas chromatography, solid-phase microextraction-gas chromatography-mass spectrometry and olfactometry. J. Chromatogr. A 2002, 976, 265-275. [CrossRef]

(C) 2018 by the authors. Licensee MDPI, Basel, Switzerland. This article is an open access article distributed under the terms and conditions of the Creative Commons Attribution (CC BY) license (http:/ / creativecommons.org/licenses/by/4.0/). 